\title{
Supplemental Material for \\ Bi-Factor Structure of the Wechsler Preschool and Primary Scale of Intelligence-Fourth Edition
}

\author{
Marley W. Watkins A. Alexander Beaujean
}

9 July 2013

\section{Contents}

\section{Contents}

1 2:6-3:11 Year-Old Group 2

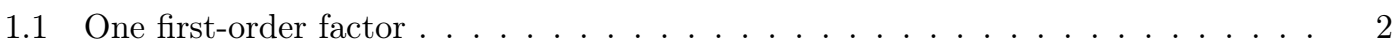

1.2 One second-order factor and two first-order factors . . . . . . . . . . . . 3

1.3 One second-order factor and three first-order factors $\ldots \ldots \ldots$. . . . . . . 4

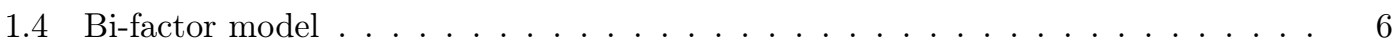

1.5 No second-order factor and three oblique first-order factors $\ldots \ldots \ldots$. . . . . 7

2 4:0-7:7 Year-Old Group $\quad 9$

2.1 One first-order factor . . . . . . . . . . . . . . . . . . . . 10

2.2 One second-order factor and two first-order factors $\ldots \ldots \ldots \ldots \ldots \ldots$

2.3 One second-order factor and three first-order factors . . . . . . . . . . . . . . . 14

2.4 One second-order factor and four first-order factors, version $1 \ldots \ldots \ldots$

2.5 One second-order factor and four first-order factors, version $2 \ldots \ldots \ldots$

2.6 One second-order factor and five first-order factors . . . . . . . . . . . . . . 20

2.7 One second-order factor, five first-order factors, and two subfactors nested within Verbal Comprehension . . . . . . . . . . . . . . . . . . 22

2.8 Bi-factor model with one Verbal Comprehension factor . . . . . . . . . . . . . . . 24

2.9 Bi-factor model with two Verbal Comprehension subfactors . . . . . . . . . . 26

2.10 No second-order factor and five oblique first-order factors . . . . . . . . . . 28

$\begin{array}{ll}\text { References } & 32\end{array}$ 
This document provides the syntax for the analyses using all the WPPSI-IV subtests as well the subtests that comprise the Primary Index scores. All data analysis was done using the $\mathbf{R}$ (version 3.0.1) statistical programming language (R Development Core Team, 2013), using the lavaan (version 0.5-13) (Rosseel, 2012) package. Below, we show the WPPSI-IV models and the lavaan syntax to fit the models using effects coding. In addition, Tables $\mathrm{C}$ and $\mathrm{F}$ show the fit statistics for each model using all the subtests as well as just the subtests used for the primary indices.

\section{2:6-3:11 Year-Old Group}

Coalson and Raiford (2012) performed factor analysis on two sets of variables. The first was with all seven subtests (Receptive Vocabulary, Picture Naming, Block Design, Object Assembly, Picture Memory, and Zoo Locations). The second only included the subtests that contribute to a primary index score, so Picture Naming was omitted. Models using the reduced dataset have $P I$ in the name, e.g, model1PI.model.

The full covariance matrix for the 2:6-3:11 year-old group comes from Coalson and Raiford (2012, p. 70) and is stored in an $\mathbf{R}$ objected named WPPSI2_3.cov for the following analyses. For an example of how to input a covariance matrix into $\mathbf{R}$ for the purposes of a confirmatory factor analysis, see Beaujean (2013).

\subsection{One first-order factor}

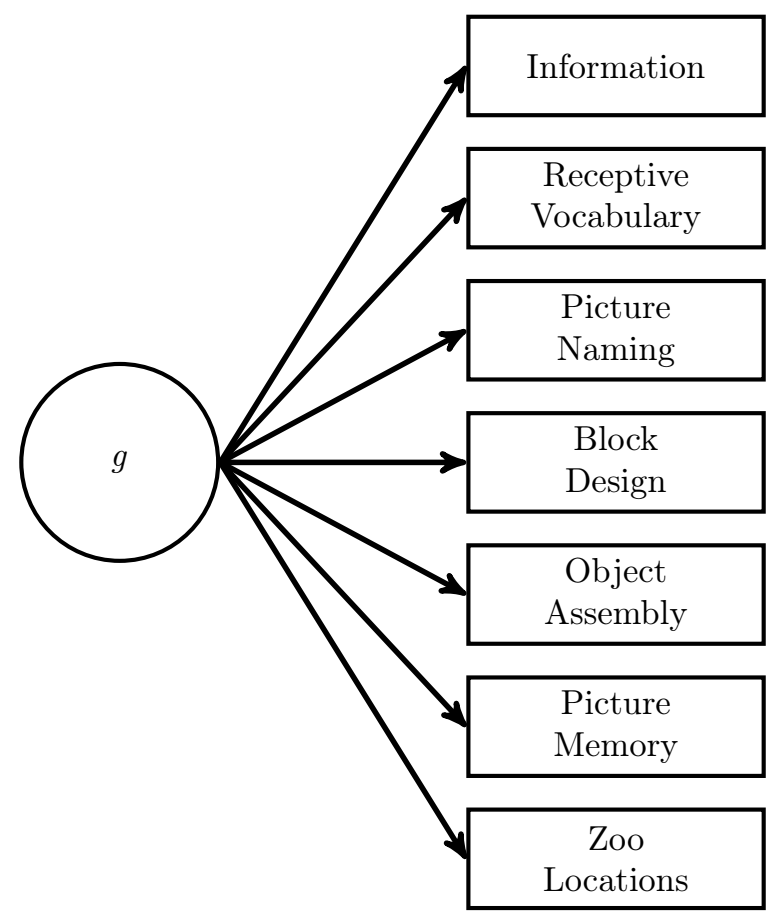

Figure 1: Model with one first-order factor for 2:6-3:11 year-old group. Residual errors not shown. 


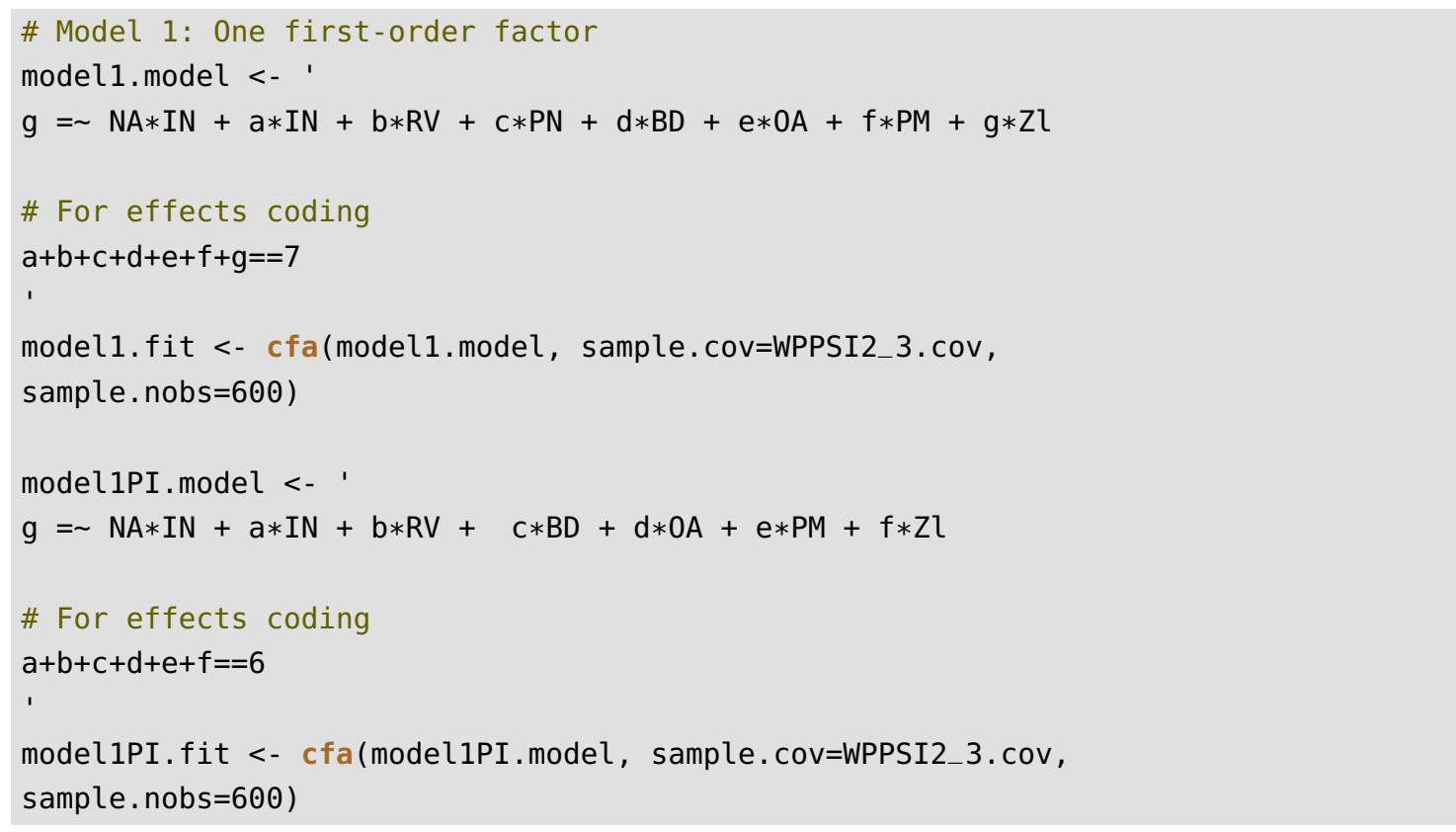

\subsection{One second-order factor and two first-order factors}

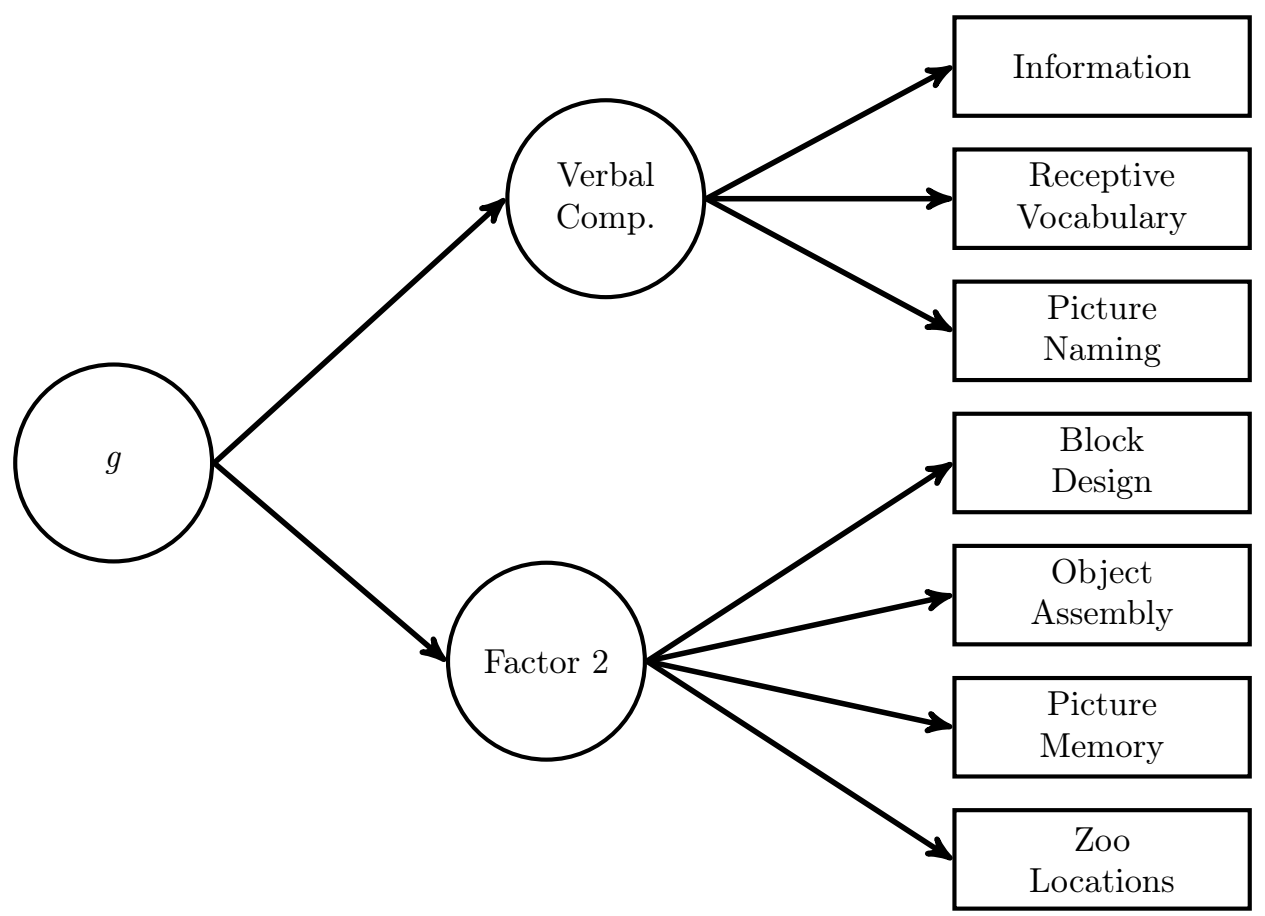

Figure 2: Model with one second-order factor and two first-order factors for 2:6-3:11 Year-Old Group. Residual errors not shown. 


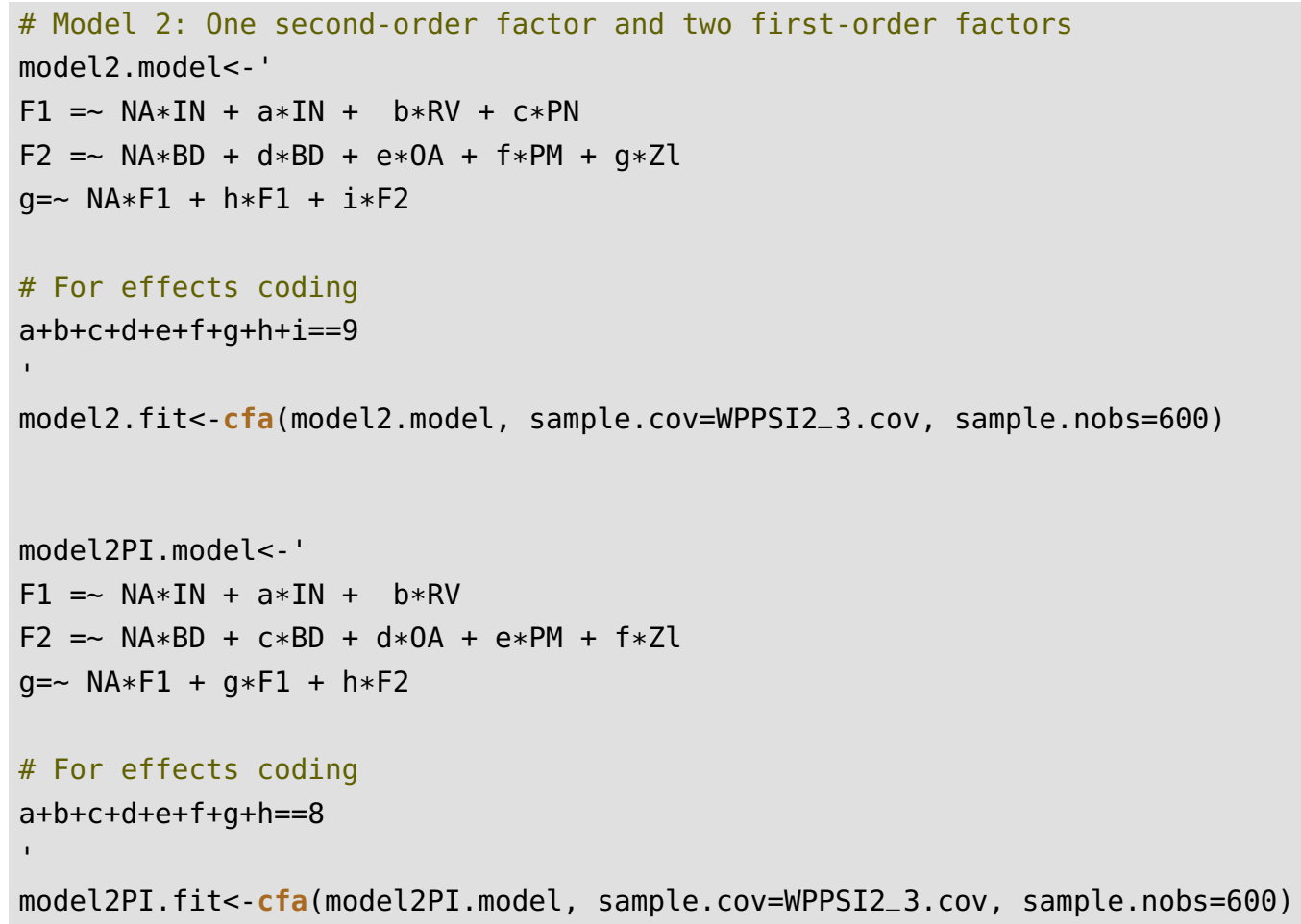

\subsection{One second-order factor and three first-order factors}

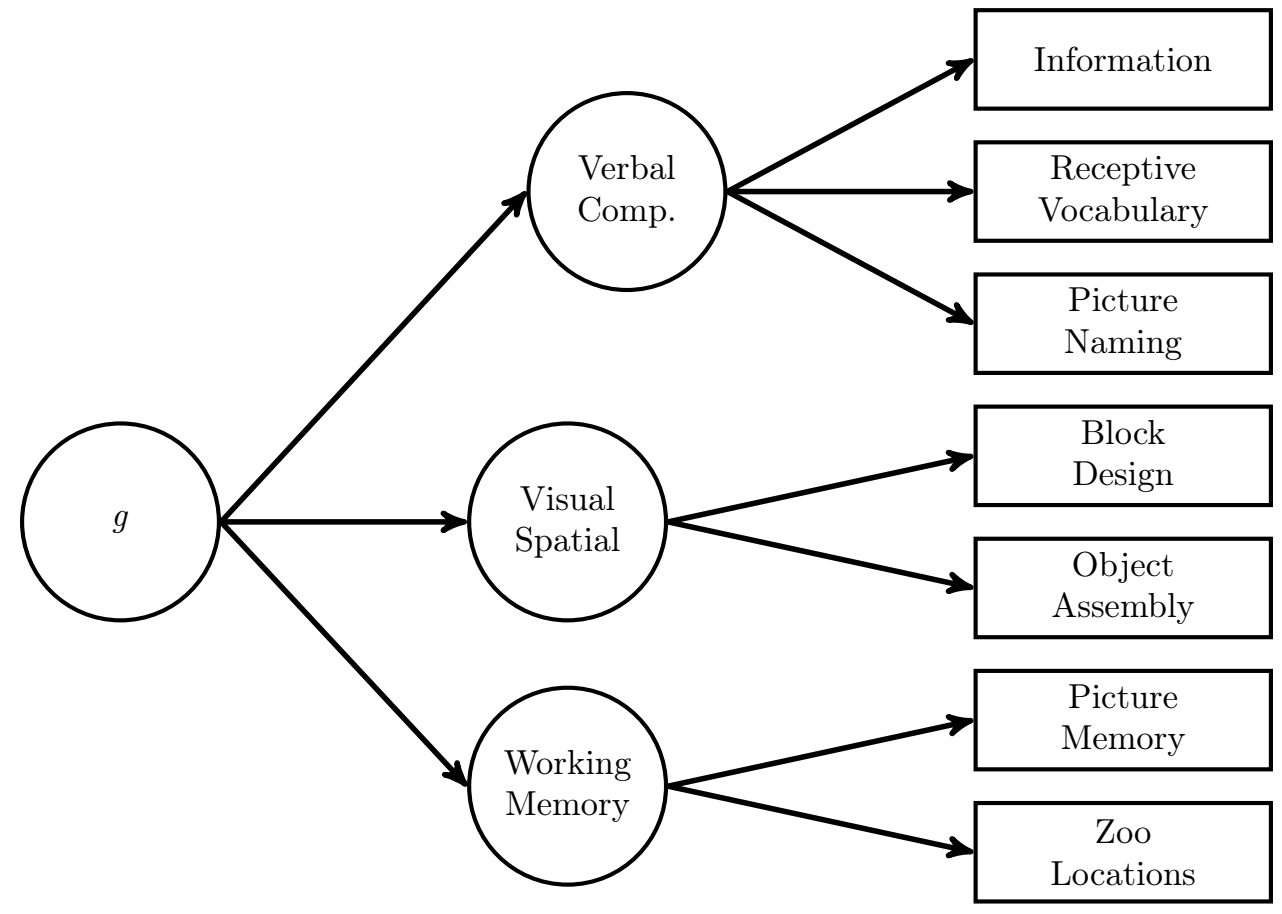

Figure 3: Model with one second-order factor and three first-order factors for 2:6-3:11 Year-Old Group. Residual errors not shown. 


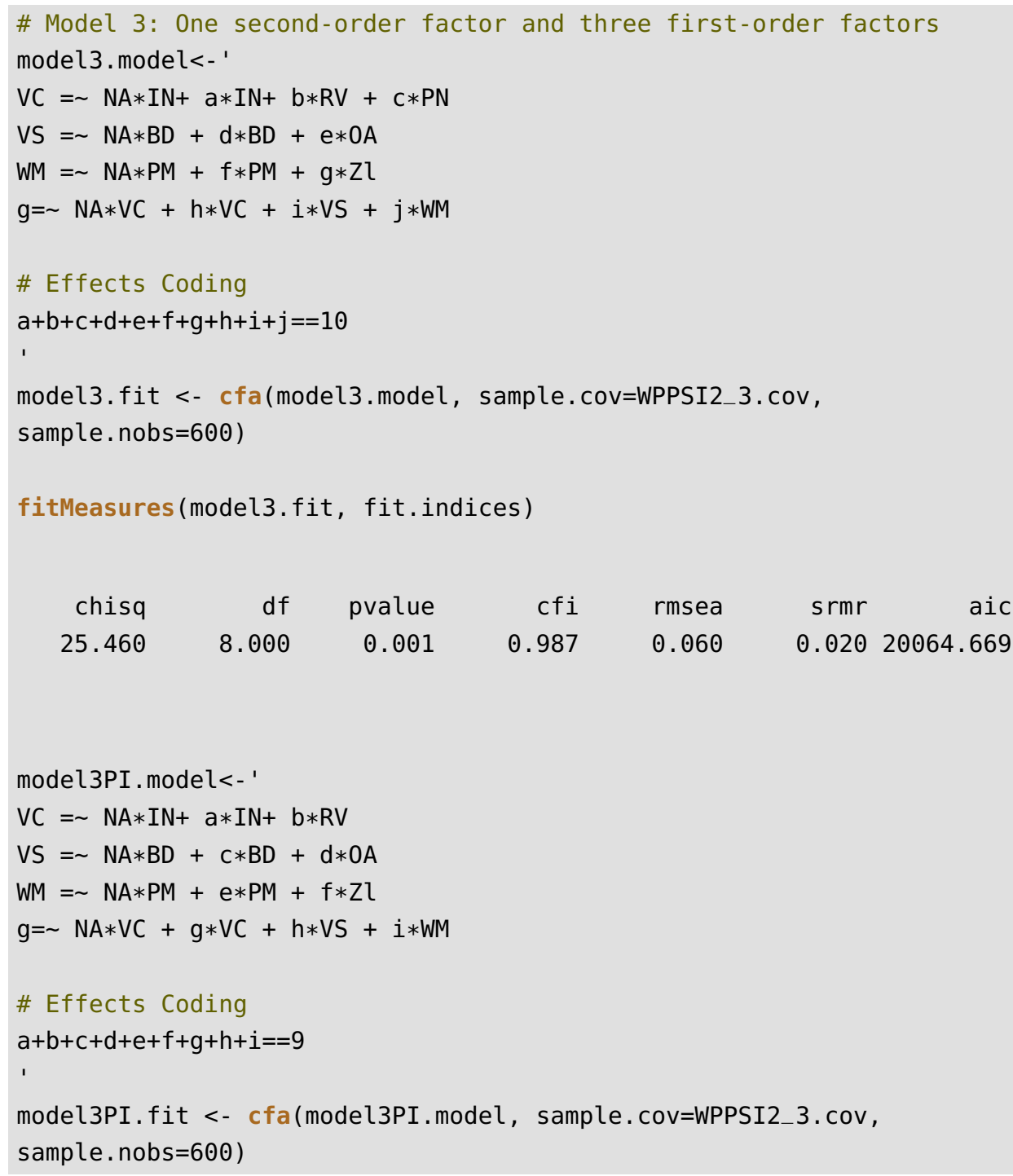




\subsection{Bi-factor model}

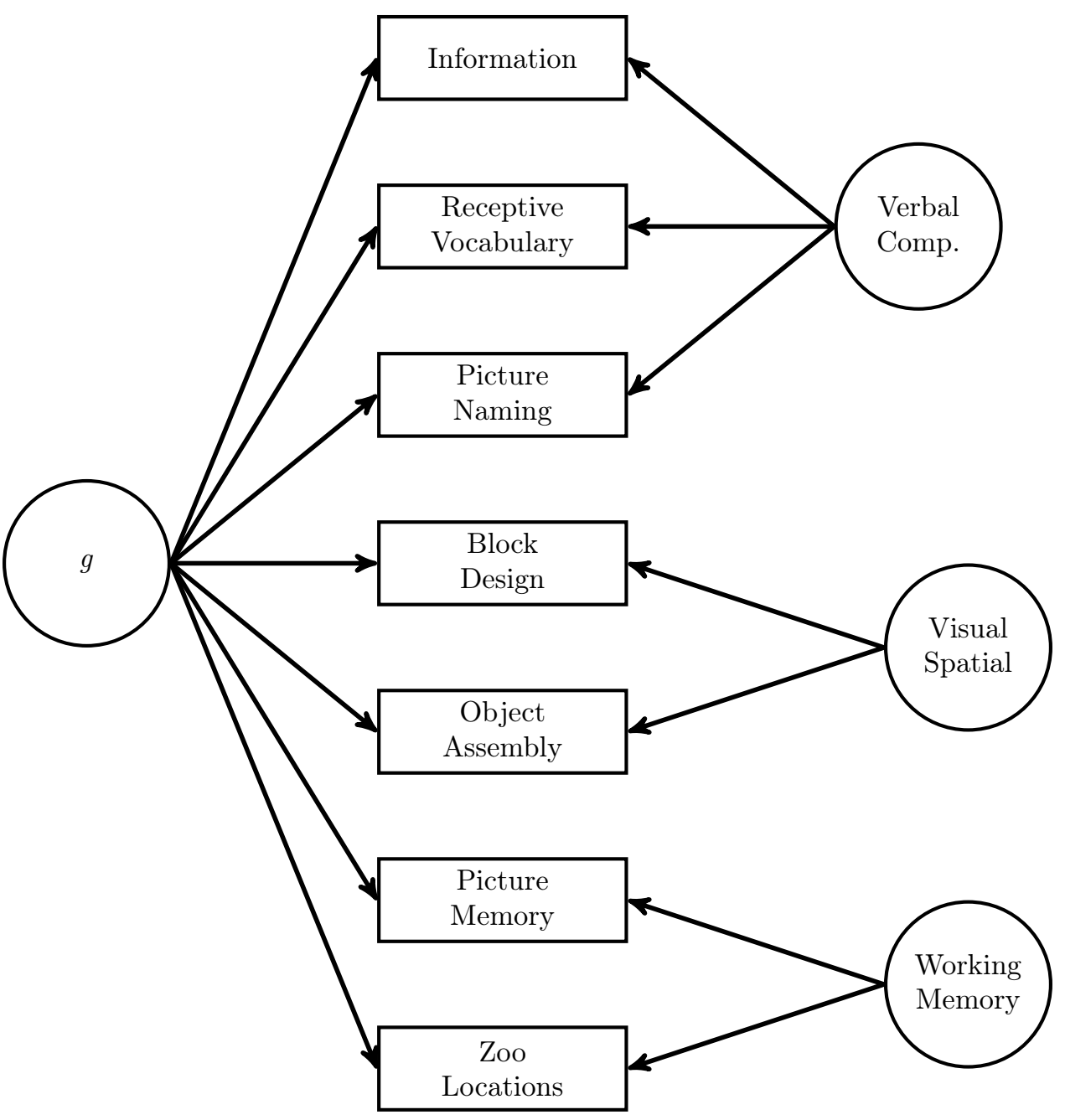

Figure 4: Bi-factor Factor Model for 2:6-3:11 year-old group. Residual errors not shown.

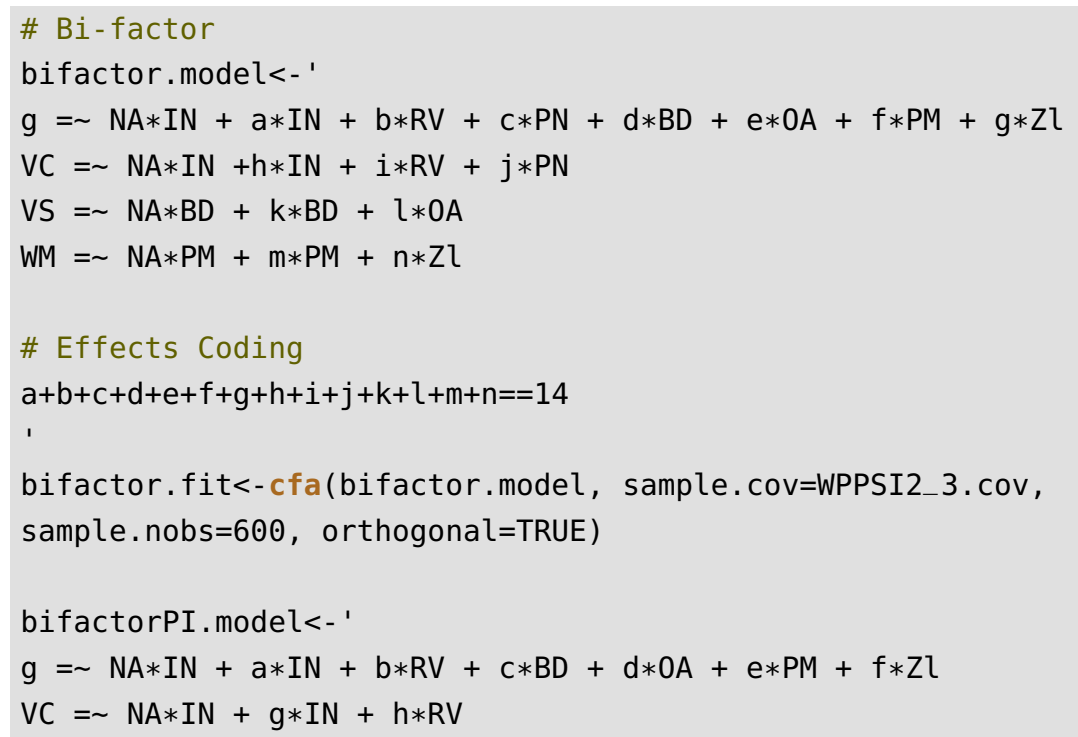




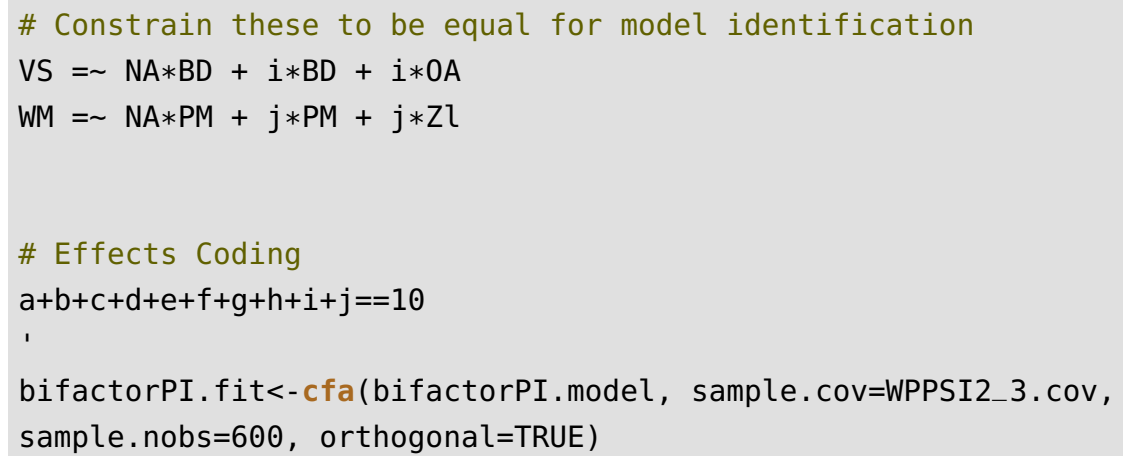

\subsection{No second-order factor and three oblique first-order factors}

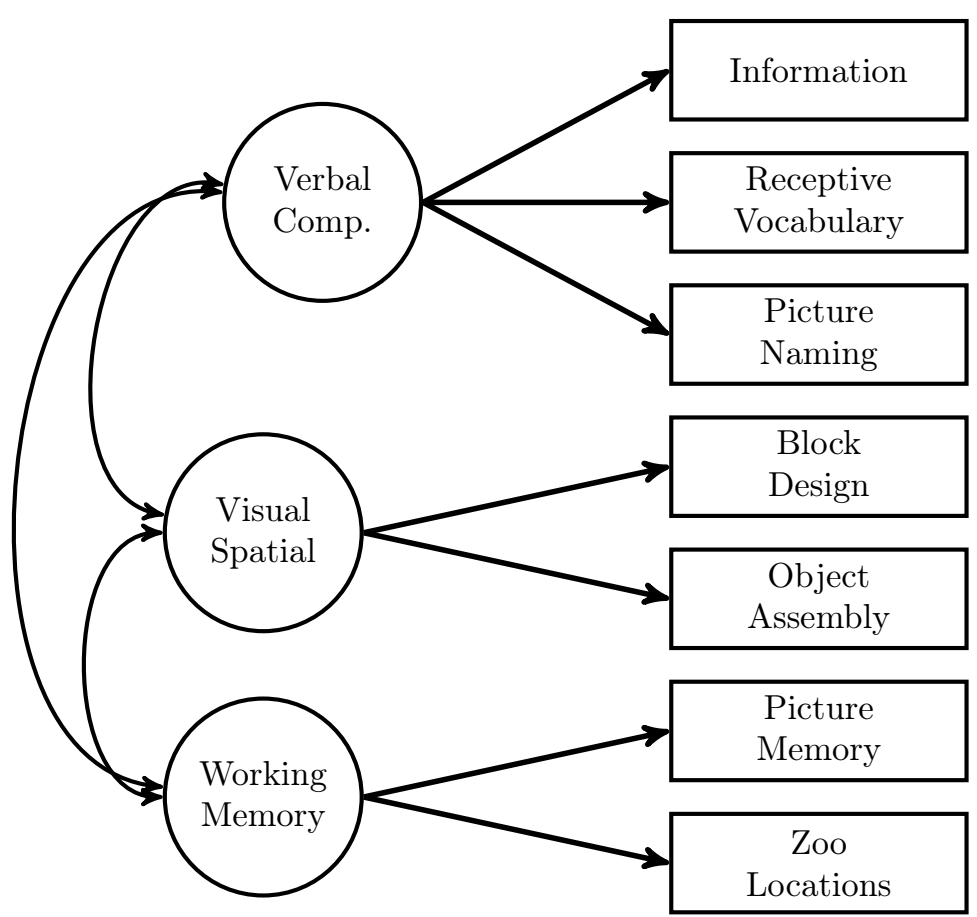

Figure 5: Model with no second-order factor and three oblique first-order factors for 2:6-3:11 year-old group. Residual errors not shown. The correlations among the factors are given in Table A and the variance explained and $\omega$ reliability estimates are given in Table B.

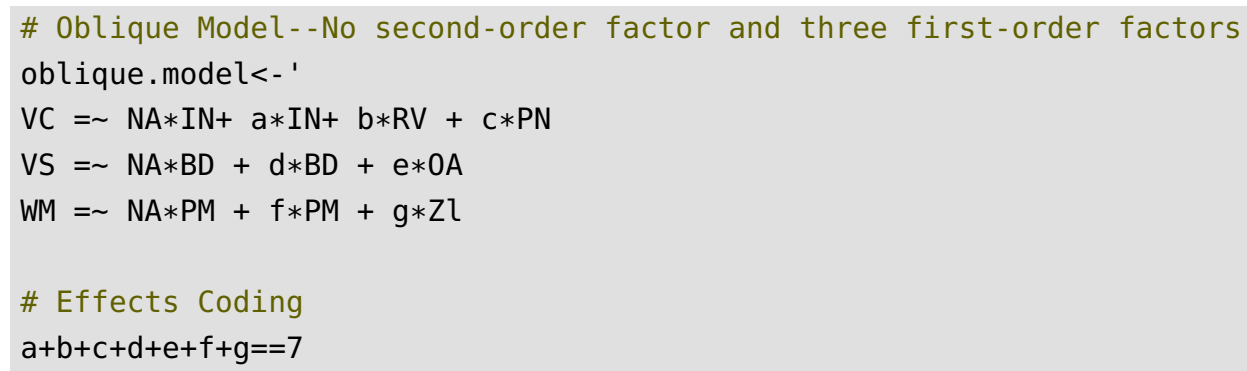




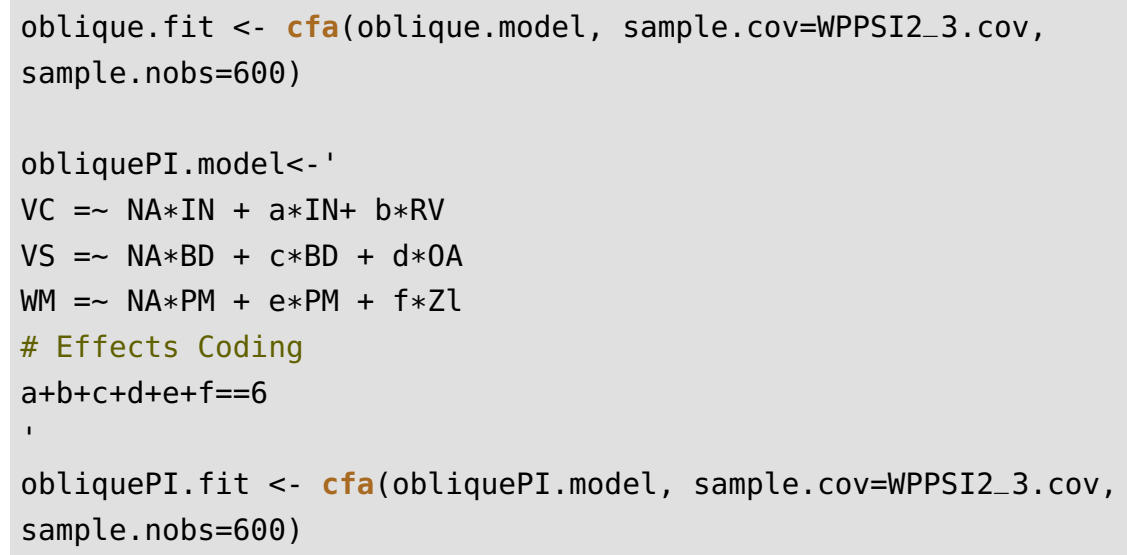

Table A: Correlations among the latent variables for the oblique model for 2:6-3:11 year-old group.

\begin{tabular}{|c|c|c|c|c|}
\hline \multicolumn{5}{|c|}{ All Subtests } \\
\hline & Subtest & 1 & 2 & 3 \\
\hline 1.00 & Verbal Comp. & 1.00 & 0.81 & 0.78 \\
\hline 2.00 & Visual Spatial & 0.81 & 1.00 & 0.85 \\
\hline 3.00 & Working Memory & 0.78 & 0.85 & 1.00 \\
\hline \multicolumn{5}{|c|}{ Primary Index Subtests } \\
\hline & Subtest & 1 & 2 & 3 \\
\hline 1.00 & Verbal Comp. & 1.00 & 0.85 & 0.84 \\
\hline 2.00 & Visual Spatial & 0.85 & 1.00 & 0.84 \\
\hline 3.00 & Working Memory & 0.84 & 0.84 & 1.00 \\
\hline
\end{tabular}

Table B: Sources of Variance in the Wechsler Preschool and Primary Scale of IntelligenceFourth Edition Among 600 Children Aged 2:6 to 3:11 Years for the Oblique Mode.

\begin{tabular}{|c|c|c|c|c|c|c|c|c|c|c|c|c|}
\hline \multirow[b]{3}{*}{ Subtest } & \multicolumn{10}{|c|}{ Ages 2:6-3:11 Years } & \multirow[b]{3}{*}{$h^{2}$} & \multirow[b]{3}{*}{$u^{2}$} \\
\hline & \multicolumn{2}{|c|}{$\mathrm{VC}$} & \multicolumn{2}{|c|}{ VS } & \multicolumn{2}{|c|}{ WM } & \multicolumn{2}{|c|}{ FR } & \multicolumn{2}{|c|}{ PS } & & \\
\hline & $b$ & Var & $b$ & Var & $b$ & Var & $b$ & Var & $b$ & Var & & \\
\hline IN & 0.82 & 67.08 & & & & & & & & & 67.08 & 32.92 \\
\hline RV & 0.72 & 52.27 & & & & & & & & & 52.27 & 47.73 \\
\hline $\mathrm{PN}$ & 0.80 & 63.20 & & & & & & & & & 63.20 & 36.80 \\
\hline $\mathrm{BD}$ & & & 0.66 & 43.69 & & & & & & & 43.69 & 56.31 \\
\hline $\mathrm{OA}$ & & & 0.61 & 36.60 & & & & & & & 36.60 & 63.40 \\
\hline PM & & & & & 0.70 & 49.28 & & & & & 49.28 & 50.72 \\
\hline ZL & & & & & 0.51 & 26.32 & & & & & 26.32 & 73.68 \\
\hline Total & & 26.1 & & 11.5 & & 10.8 & & & & & & \\
\hline$\omega$ & & 0.82 & & 0.57 & & 0.54 & & & & & & \\
\hline
\end{tabular}

Note. $b=$ standardized factor loading, Var $=\%$ variance explained, $h^{2}=$ communality, $u^{2}=$ uniqueness, $\mathrm{VC}=$ Verbal Comprehension factor, $\mathrm{VS}=$ Visual-Spatial factor, $\mathrm{WM}=$ Working Memory factor, PS = Processing Speed factor, FR = Fluid Reasoning factor, $\mathrm{IN}=$ Information, $\mathrm{RV}=$ Receptive Vocabulary, $\mathrm{PN}=$ Picture Naming, $\mathrm{BD}=$ Block Design, $\mathrm{OA}=$ Object Assembly, $\mathrm{PM}=$ Picture Memory, $\mathrm{ZL}=$ Zoo Locations, $\omega=$ omega. $\omega$ estimated from the omega software program (Watkins, 2013). 
Table C: Fit Statistics for the models used for the 2:6-3:11 year-old group data.

\begin{tabular}{lccccccc}
\hline Model & $\chi^{2}$ & $d f$ & $p$ & CFI & RMSEA & SRMR & AIC \\
\hline \multicolumn{7}{c}{ All Subtests } \\
\hline Model 1 & 70.17 & 14.00 & 0.00 & 0.96 & 0.08 & 0.04 & 20097.37 \\
Model 2 & 31.46 & 10.00 & 0.00 & 0.98 & 0.06 & 0.02 & 20066.66 \\
Model 3 & 25.46 & 8.00 & 0.00 & 0.99 & 0.06 & 0.02 & 20064.67 \\
Bifactor & 13.73 & 4.00 & 0.01 & 0.99 & 0.06 & 0.01 & 20060.94 \\
Oblique & 25.46 & 9.00 & 0.00 & 0.99 & 0.06 & 0.02 & 20062.67 \\
\hline \multicolumn{7}{c}{ Primary Index Subtests } \\
\hline Model 1 & 22.19 & 9.00 & 0.01 & 0.99 & 0.05 & 0.03 & 17467.20 \\
Model 2 & 9.82 & 5.00 & 0.08 & 0.99 & 0.04 & 0.02 & 17462.83 \\
Model 3 & 2.92 & 3.00 & 0.40 & 1.00 & 0.00 & 0.01 & 17459.93 \\
Bifactor & 2.92 & 2.00 & 0.23 & 1.00 & 0.03 & 0.01 & 17461.93 \\
Oblique & 2.92 & 4.00 & 0.57 & 1.00 & 0.00 & 0.01 & 17457.93 \\
\hline
\end{tabular}

CFI: comparative fit index; RMSEA: root mean square error of approximation; SRMR: standardized root mean square residual; AIC: Akaike's information criterion.

\section{4:0-7:7 Year-Old Group}

As with the 2:6-3:11 year-old group, Coalson and Raiford (2012) performed factor analysis on two sets of variables for the 4:0-7:7 year old group. The first was with all fifteen subtests (Information, Similarities, Vocabulary, Comprehension, Receptive Vocabulary, Picture Naming, Block Design, Object Assembly, Matrix Reasoning, Picture Concepts, Picture Memory, Zoo Locations, Bug Search, Cancellation, and Animal Coding). The second only included the subtests that contribute to a primary index score, so Vocabulary, Comprehension, Receptive Vocabulary, Picture Naming, and Animal Coding were omitted. In addition, the for reduced data, models with subfactors within the Verbal Comprehension factor are not estimable. Models using the reduced dataset have $P I$ in the name, e.g, model1PI.model.

The full covariance matrix for the 4:0-7:7 year-old group comes from Coalson and Raiford (2012, p. 71) and is stored in an $\mathbf{R}$ objected named WPPSI4_7.cov for the following analyses. For an example of how to input a covariance matrix into $\mathbf{R}$ for the purposes of a confirmatory factor analysis, see Beaujean (2013). 


\subsection{One first-order factor}

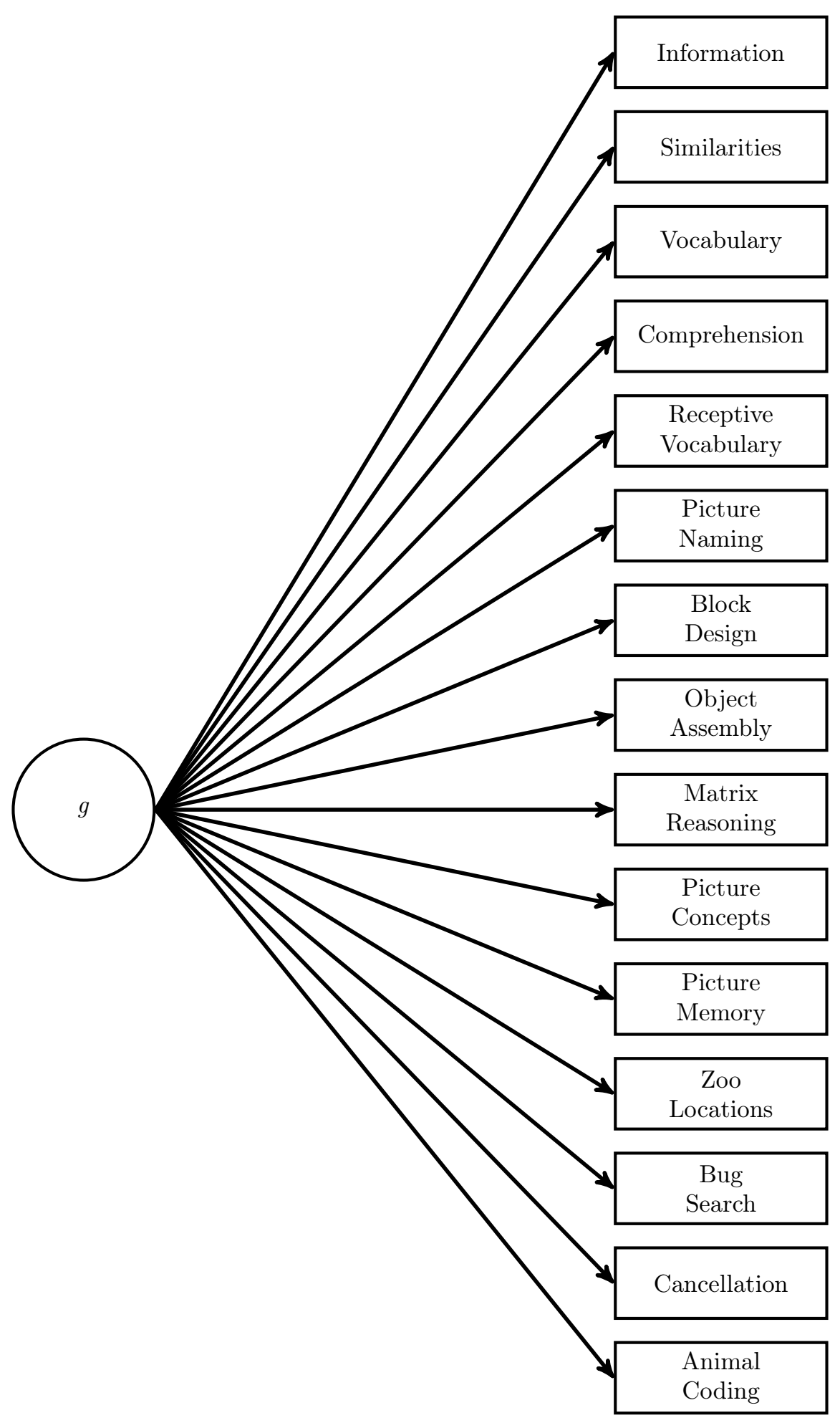

Figure 6: Single first-order factor model for 4:0-7:7 year-old group. Residual errors not shown. 


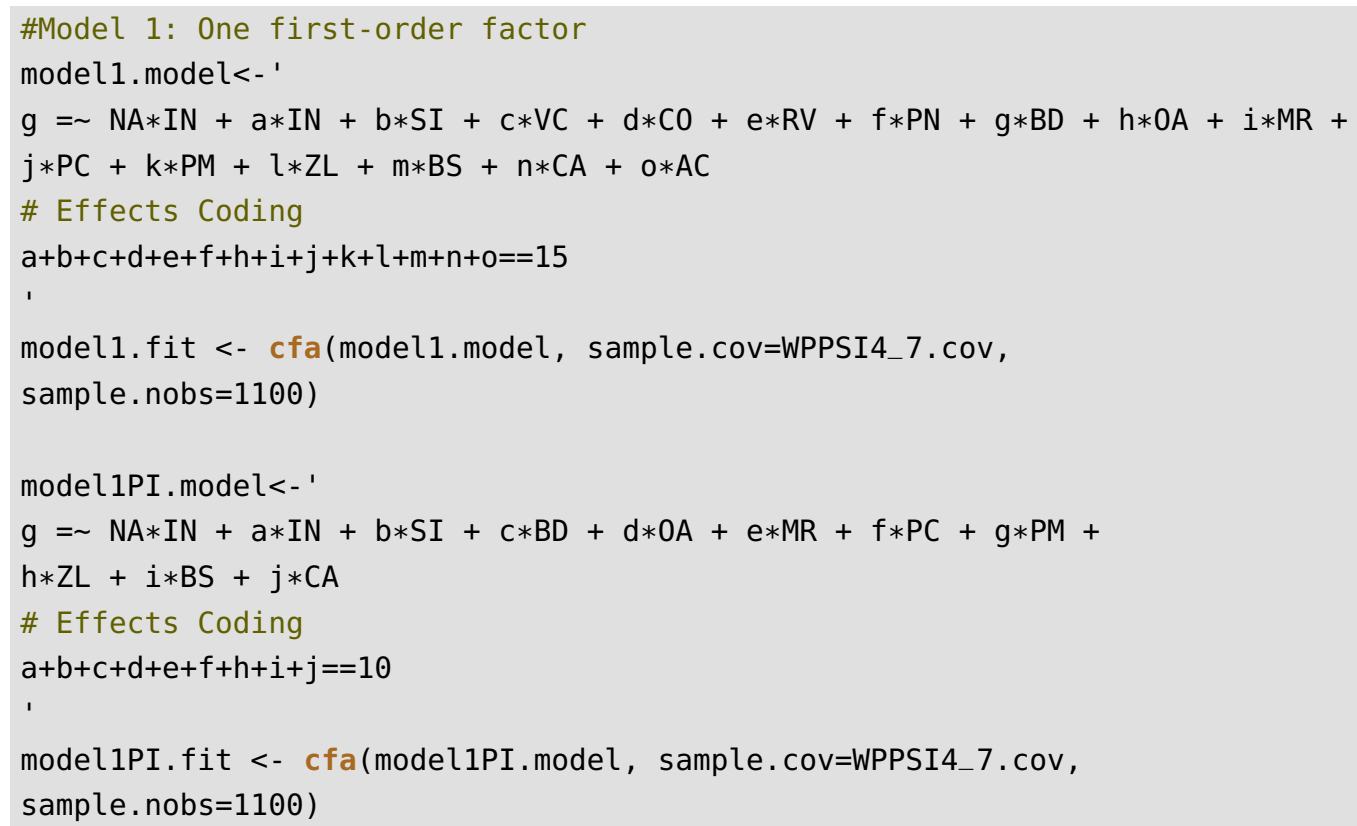




\subsection{One second-order factor and two first-order factors}

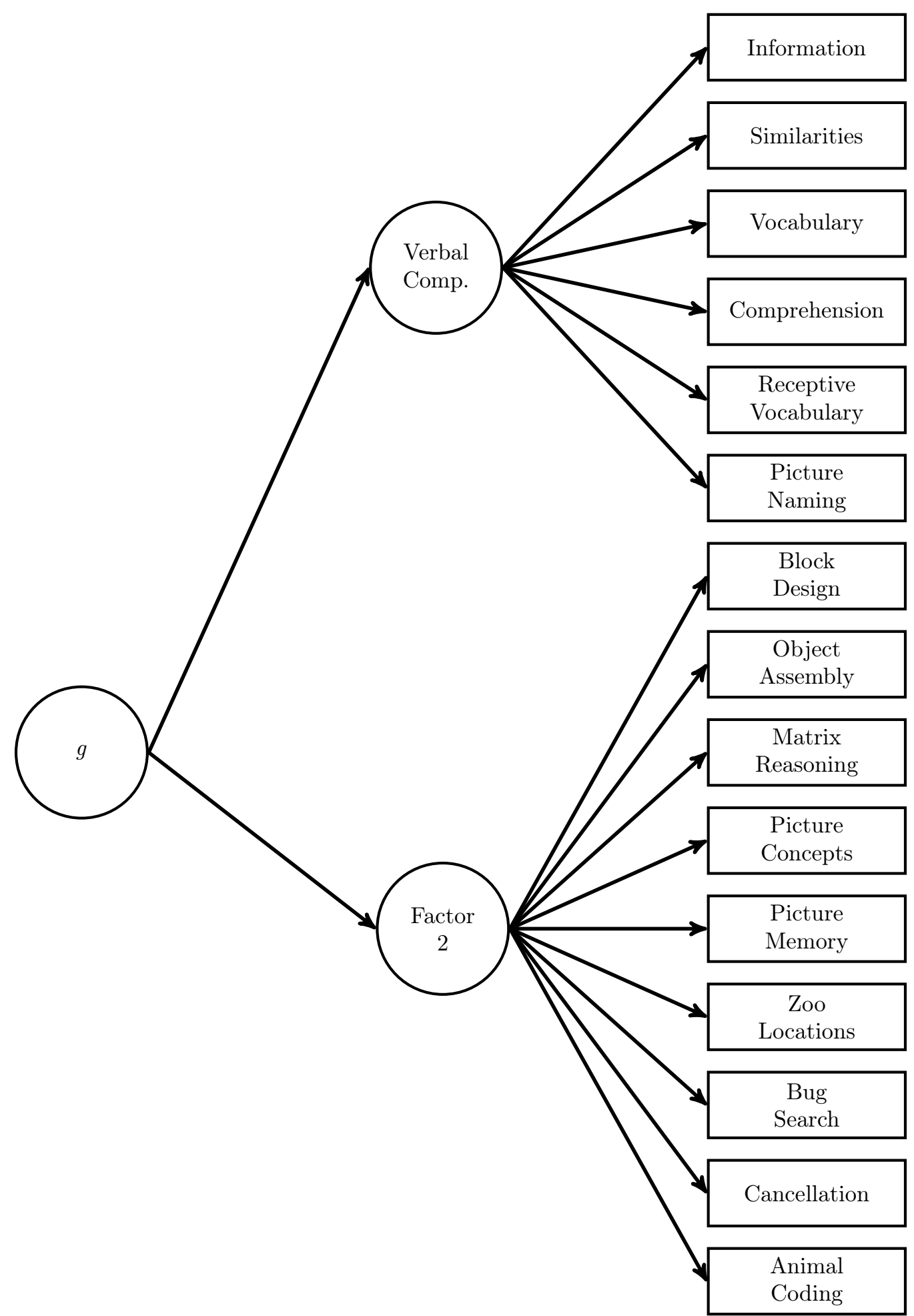

Figure 7: Model with one second-order factor and two first-order factors for 4:0-7:7 year-old group. Residual errors not shown. 


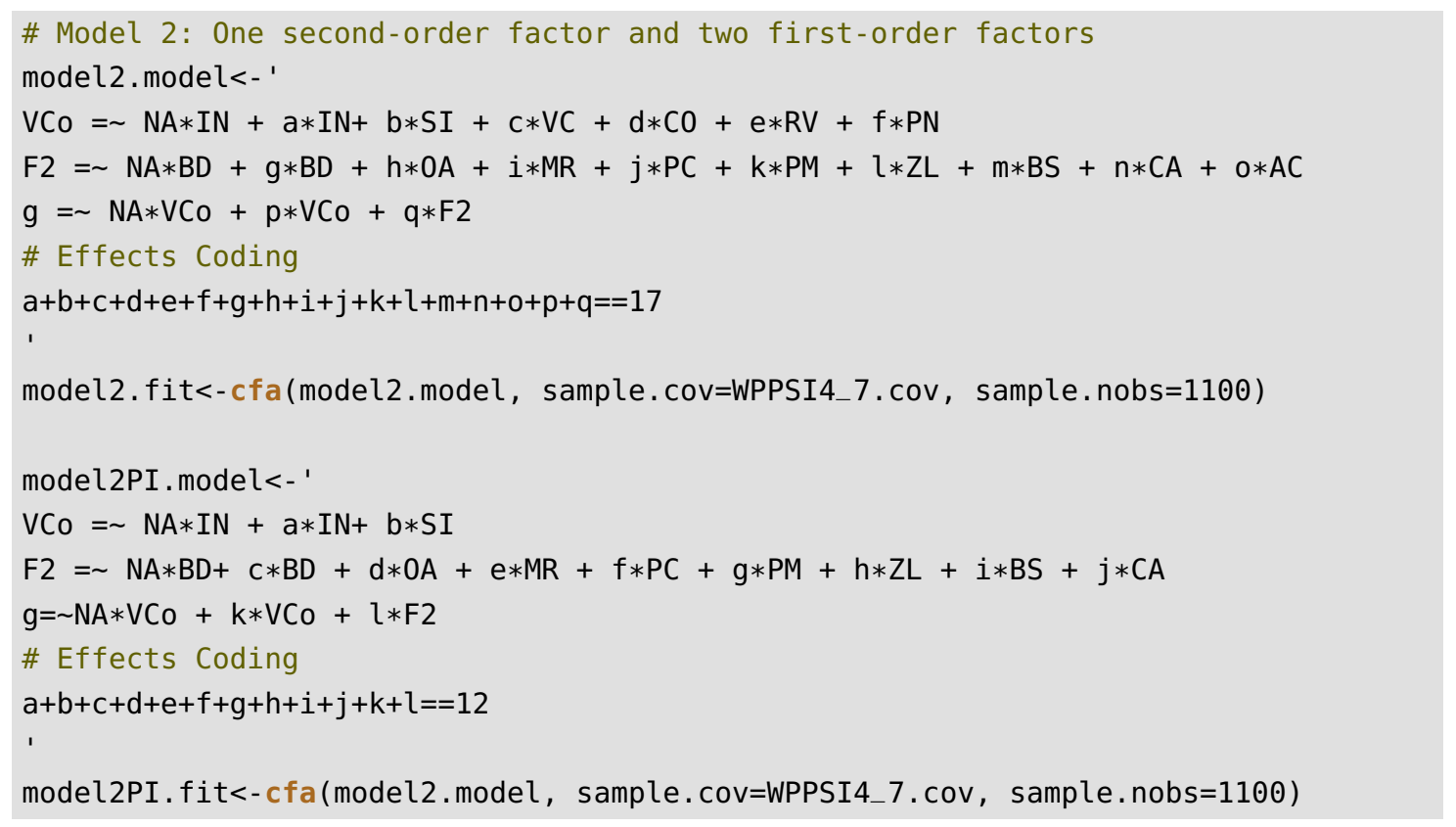




\subsection{One second-order factor and three first-order factors}

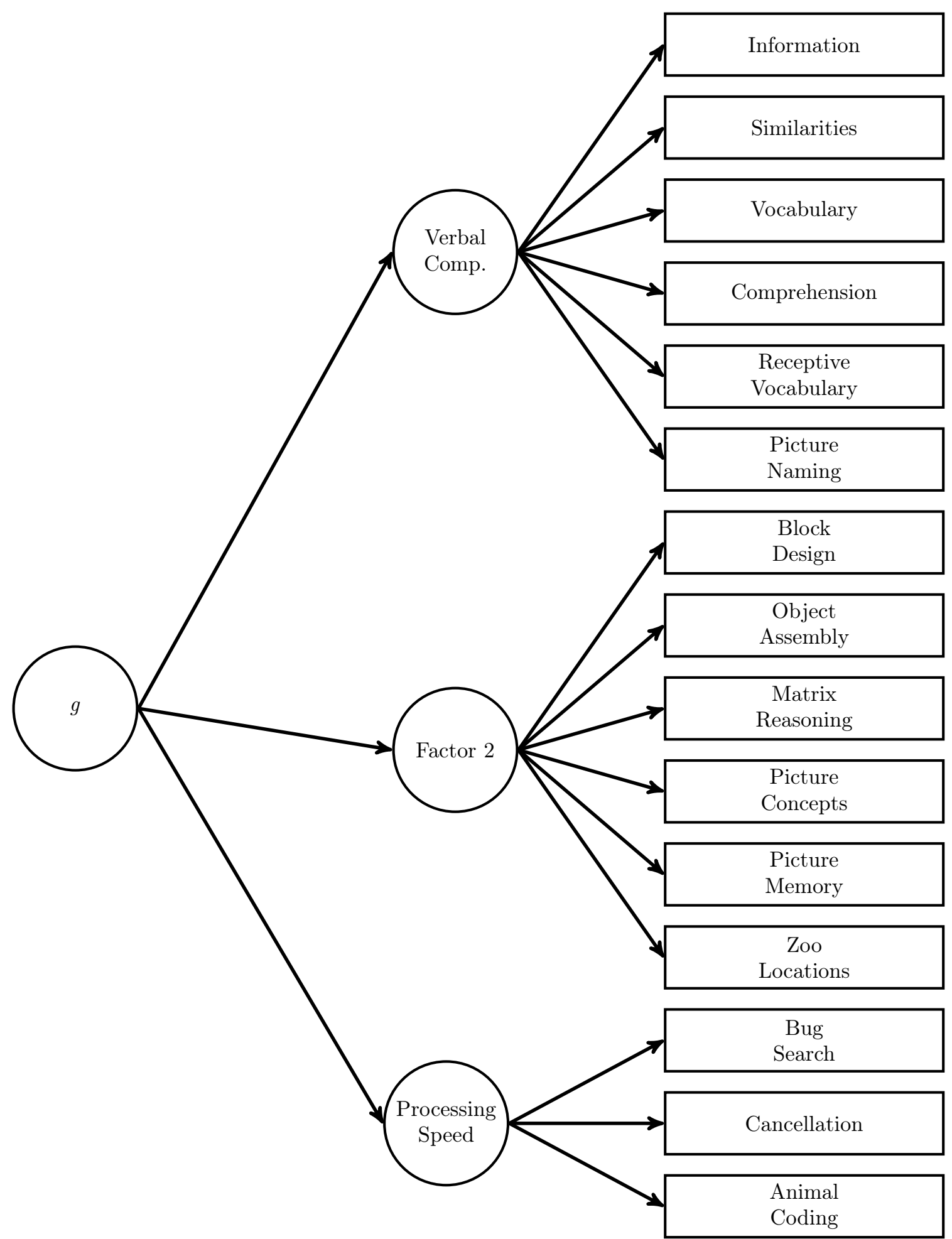

Figure 8: Model with one second-order factor and three first-order factors for 4:0-7:7 year-old group. Residual errors not shown. 


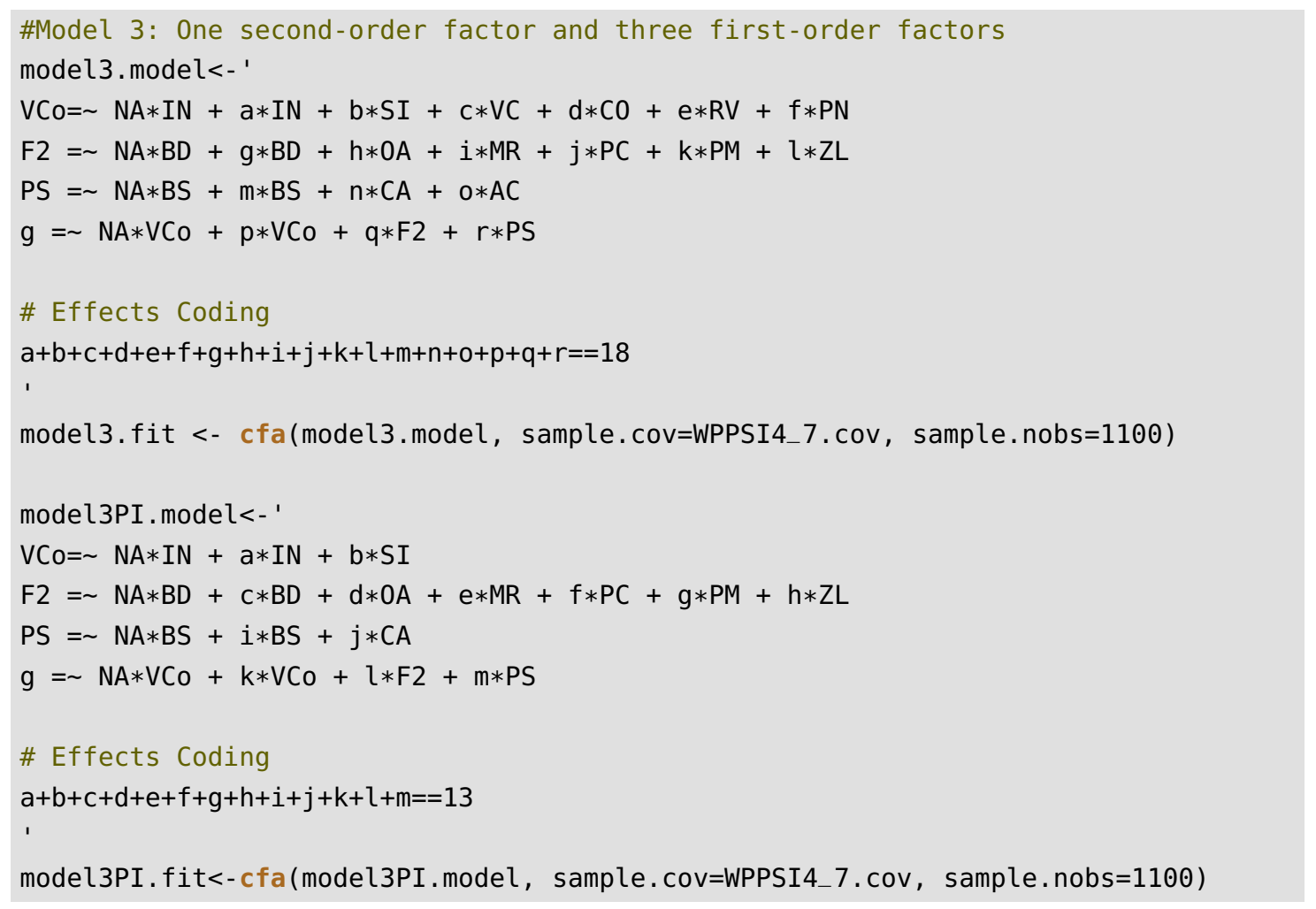




\subsection{One second-order factor and four first-order factors, version 1}

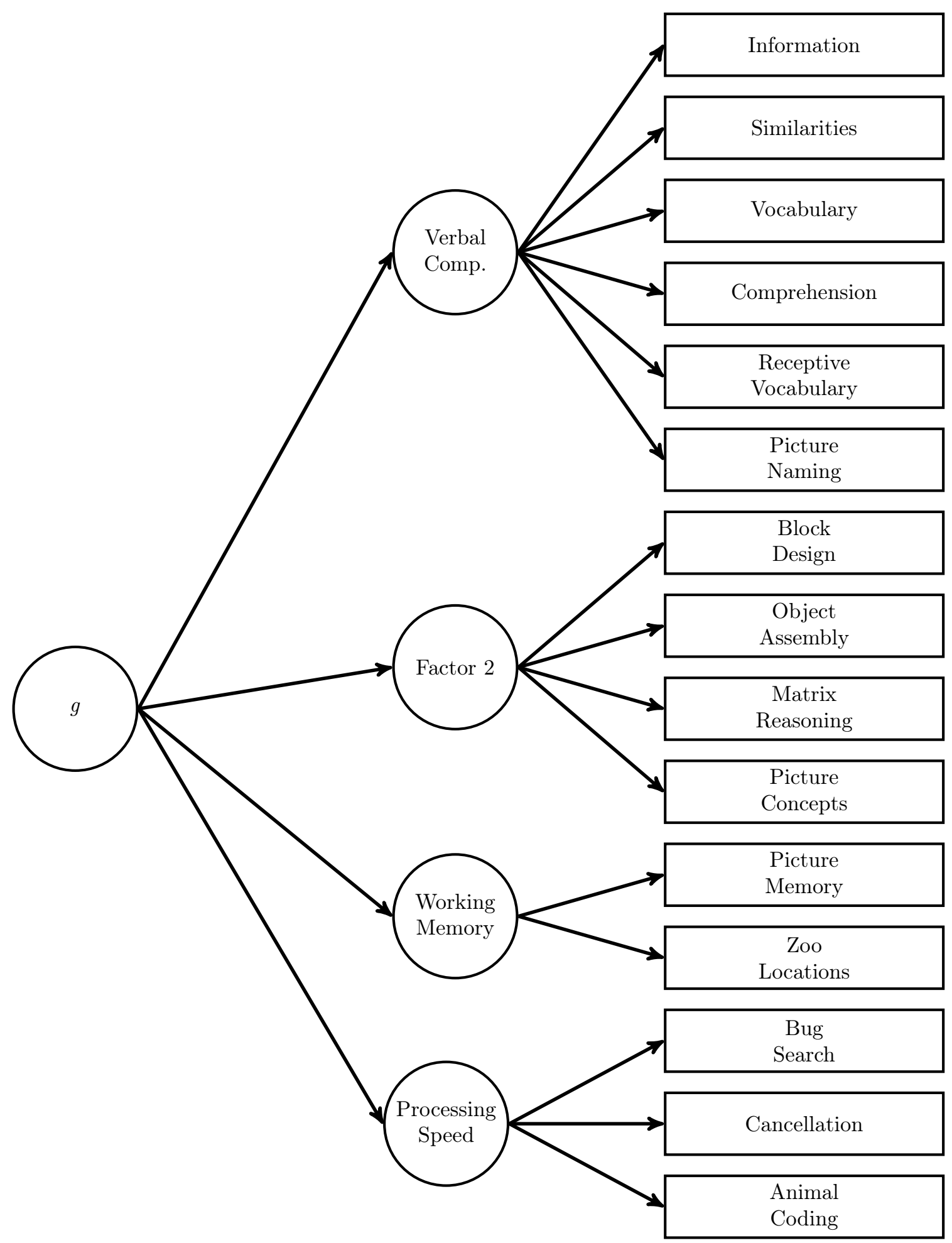

Figure 9: Model with one second-order factor and four first-order factors (version 1) for 4:0-7:7 year-old group. Residual errors not shown. 


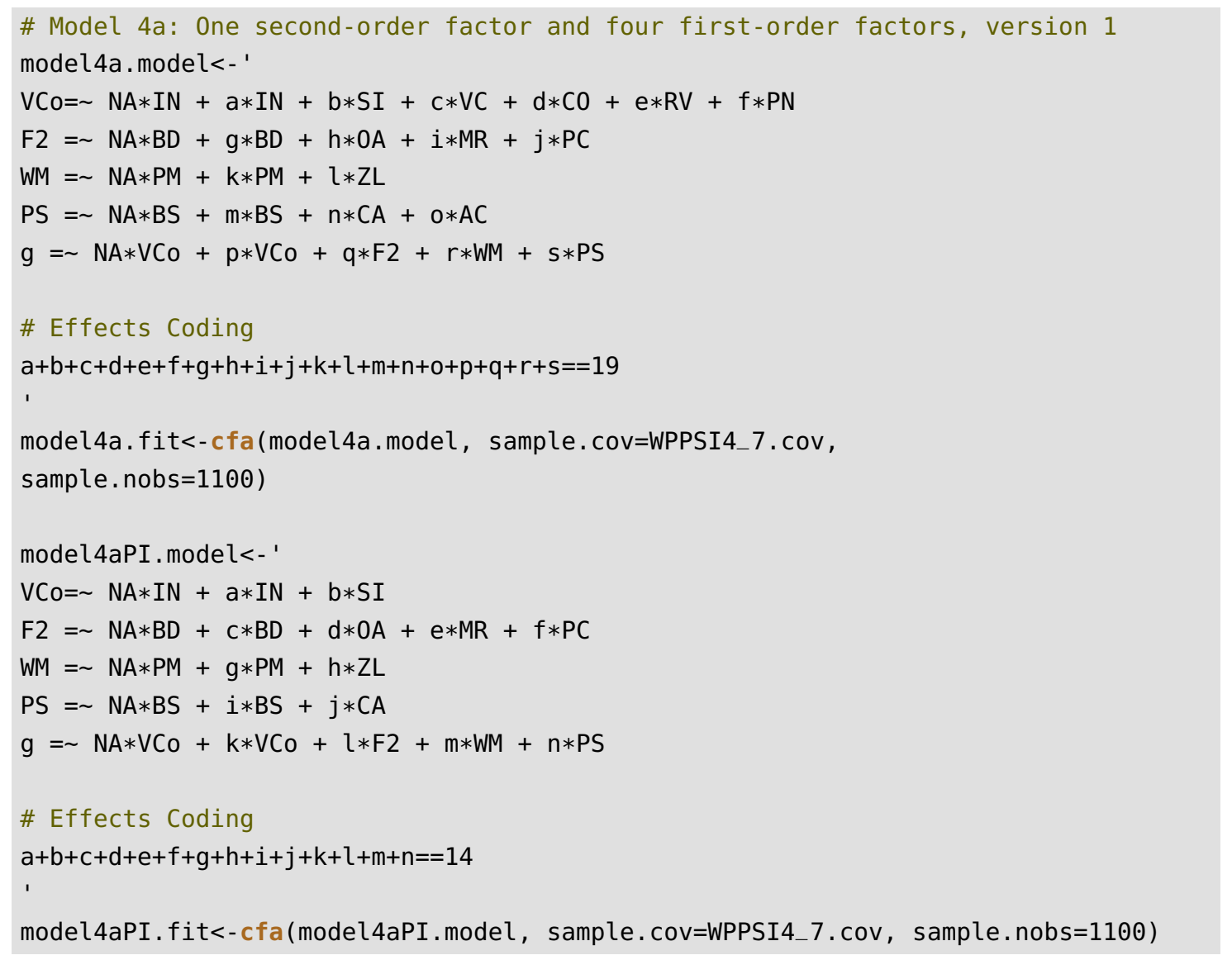




\subsection{One second-order factor and four first-order factors, version 2}

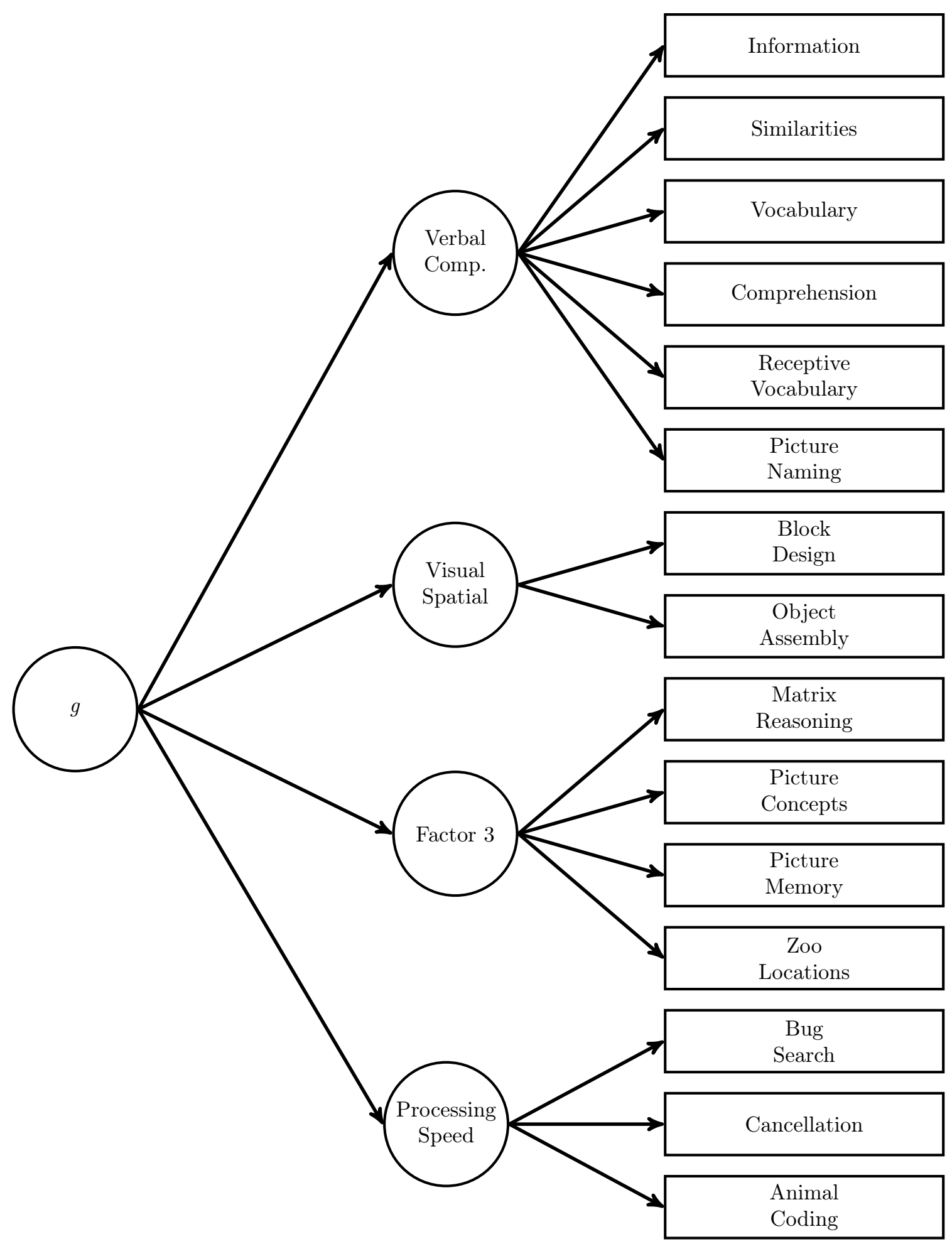

Figure 10: Model with one second-order factor and four first-order factors (version 2) for 4:0-7:7 year-old group. Residual errors not shown. 


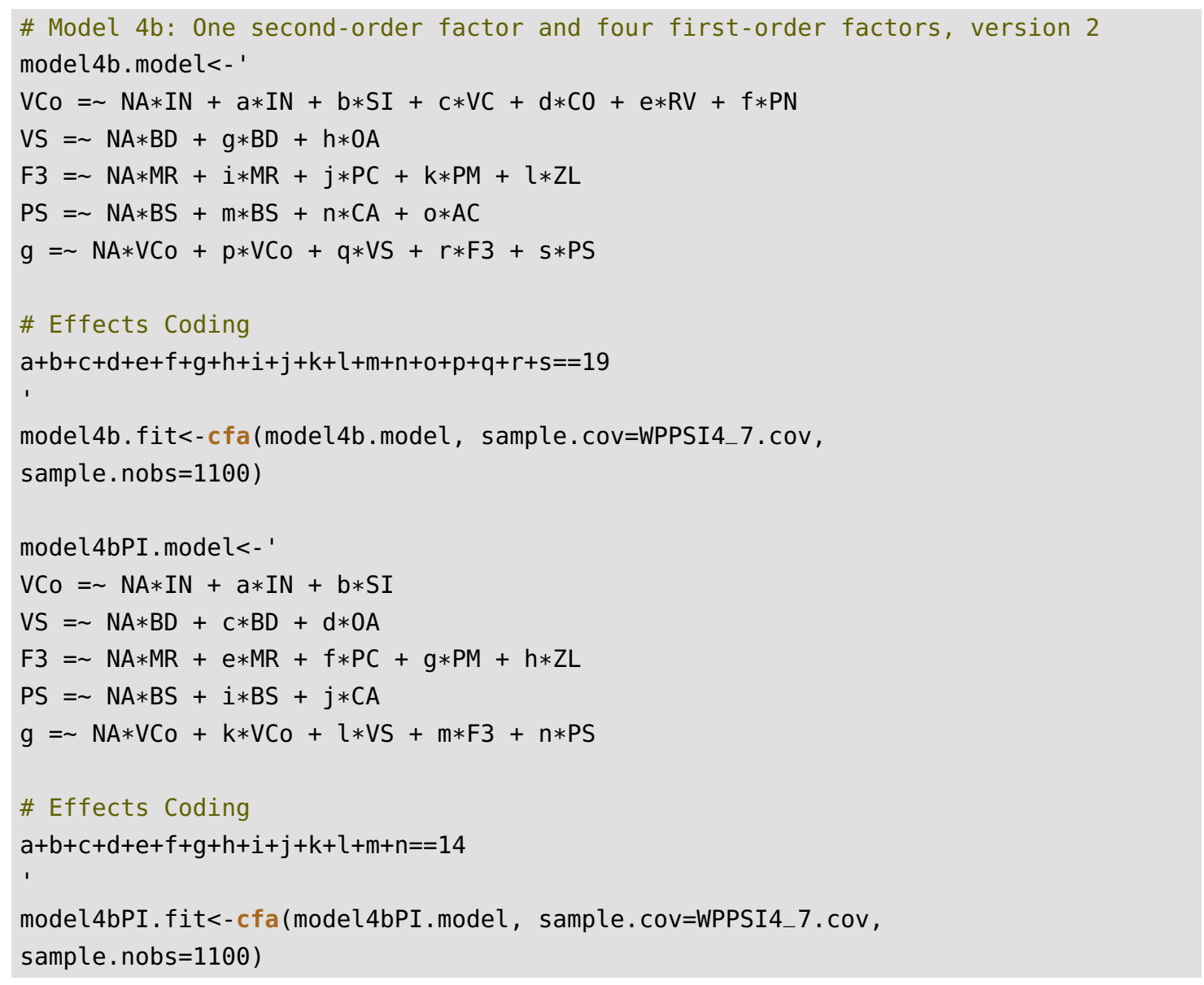




\subsection{One second-order factor and five first-order factors}

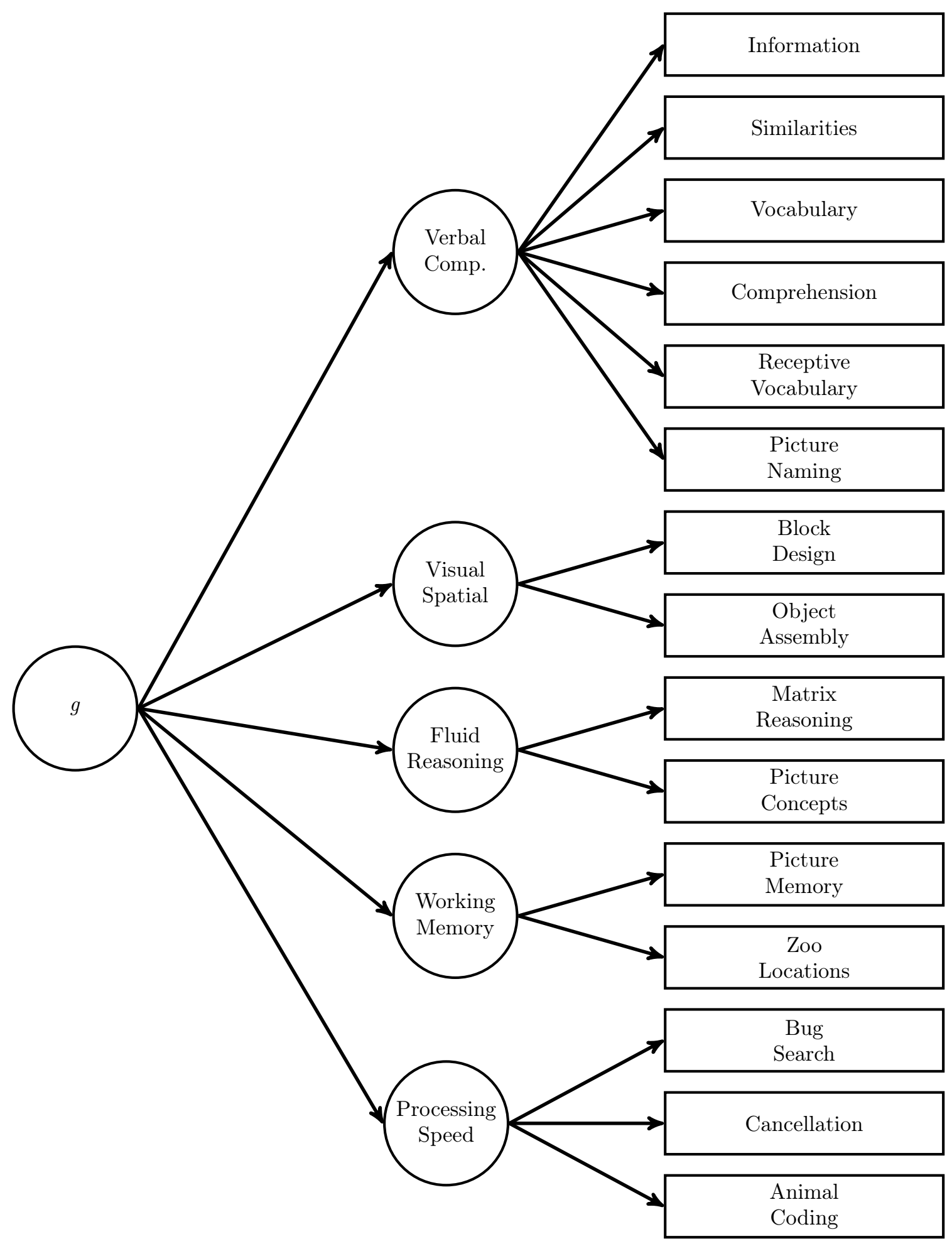

Figure 11: Model with one second-order factor and five first-order factors for 4:0-7:7 year-old group. Residual errors not shown. 


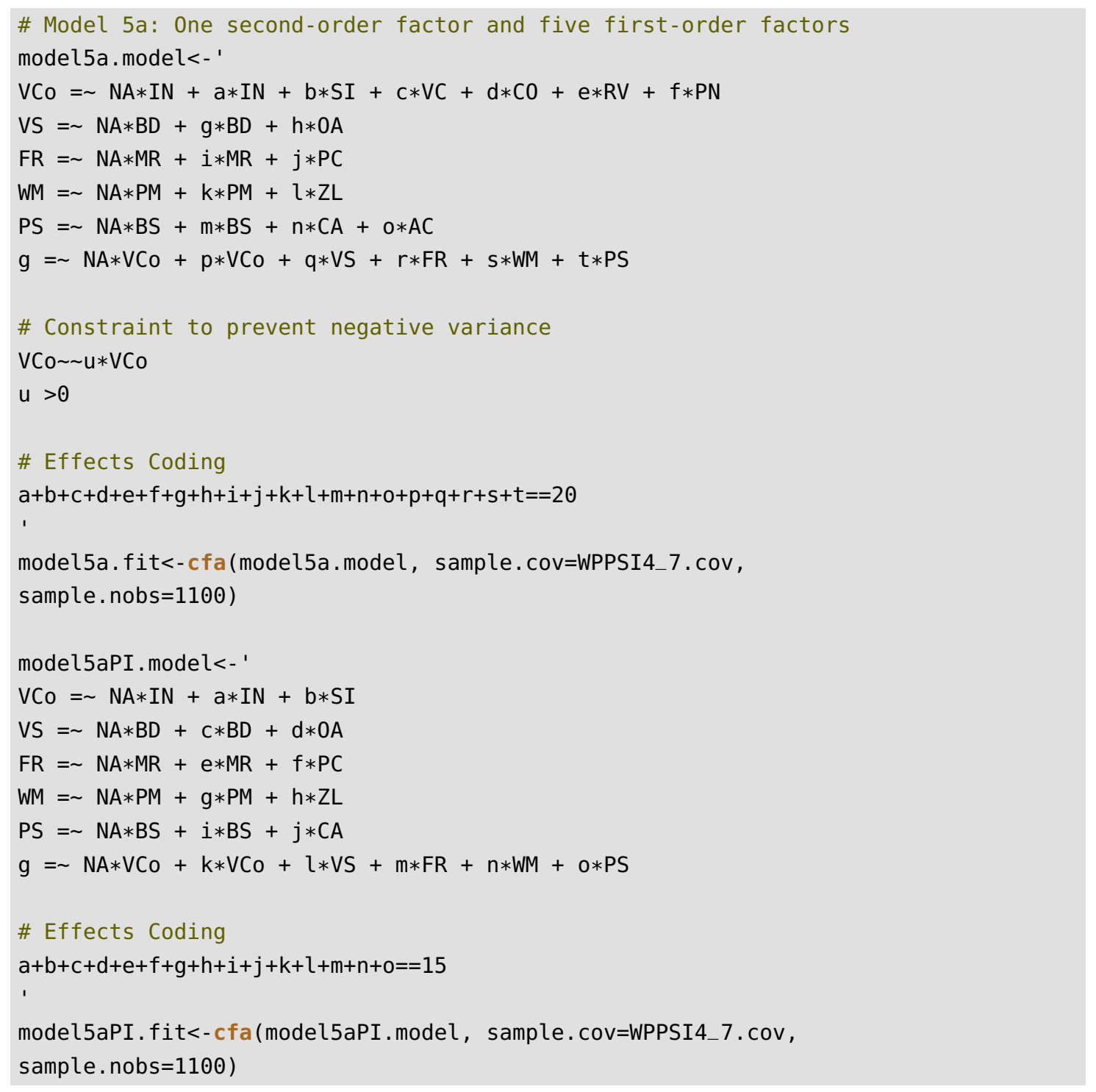


2.7 One second-order factor, five first-order factors, and two subfactors nested within Verbal Comprehension

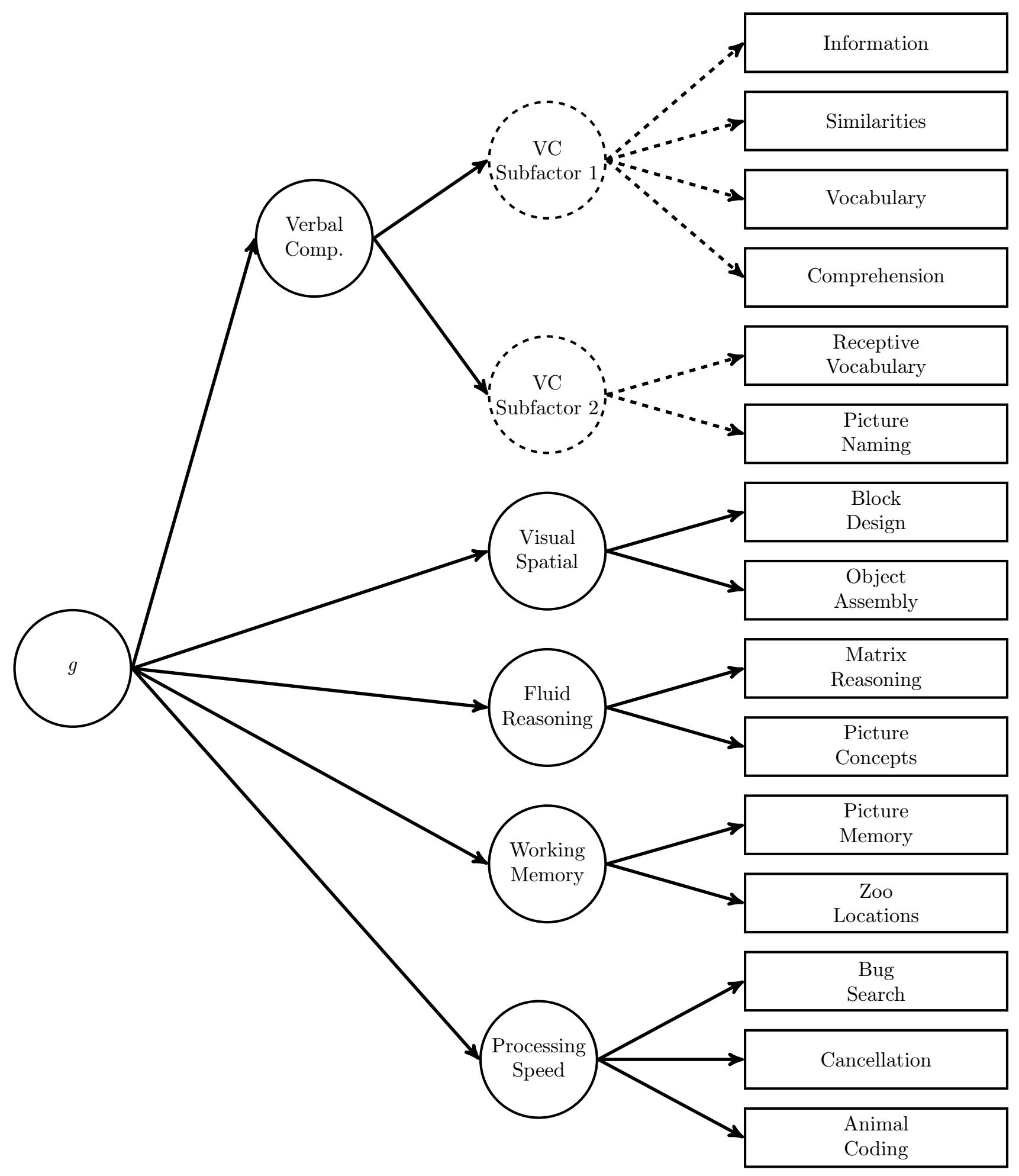

Figure 12: Model with one second-order factor, five first-order factors, and two subfactors nested within Verbal Comprehension for 4:0-7:7 year-old group. Residual errors not shown. 
2.7 One second-order factor, five first-order factors, and two subfactors nested within Verbal

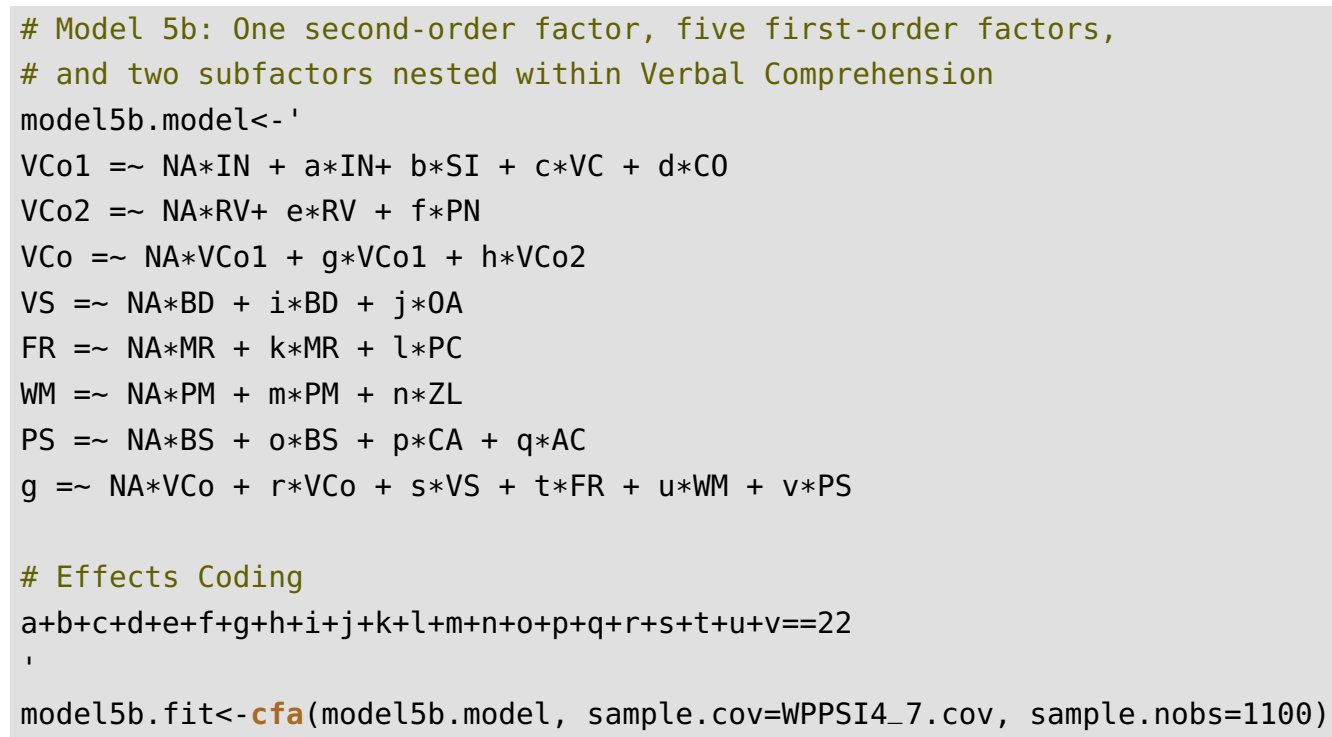




\subsection{Bi-factor model with one Verbal Comprehension factor}

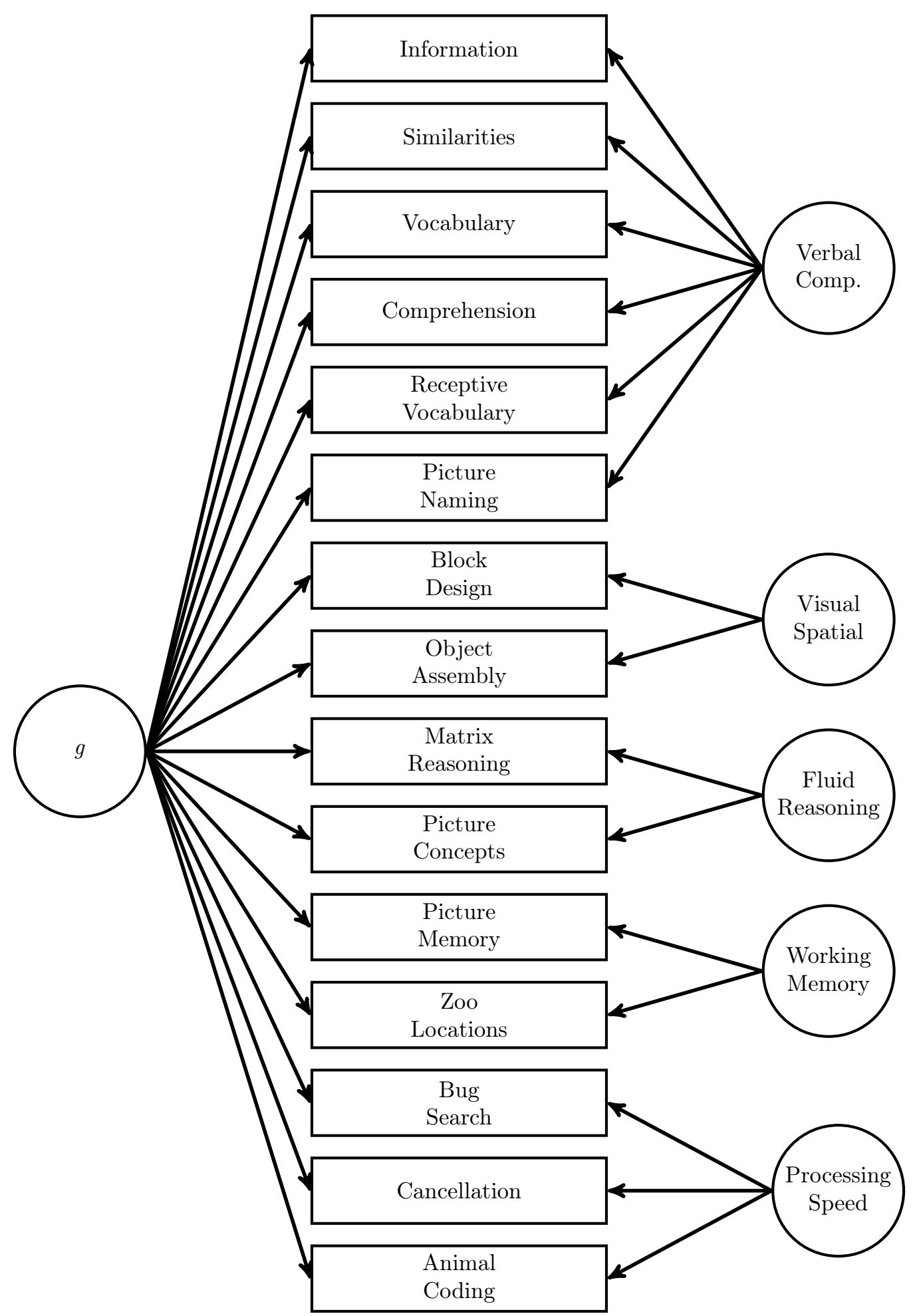

Figure 13: Bi-factor model 1 for 4:0-7:7 year-old group. Residual errors not shown. 


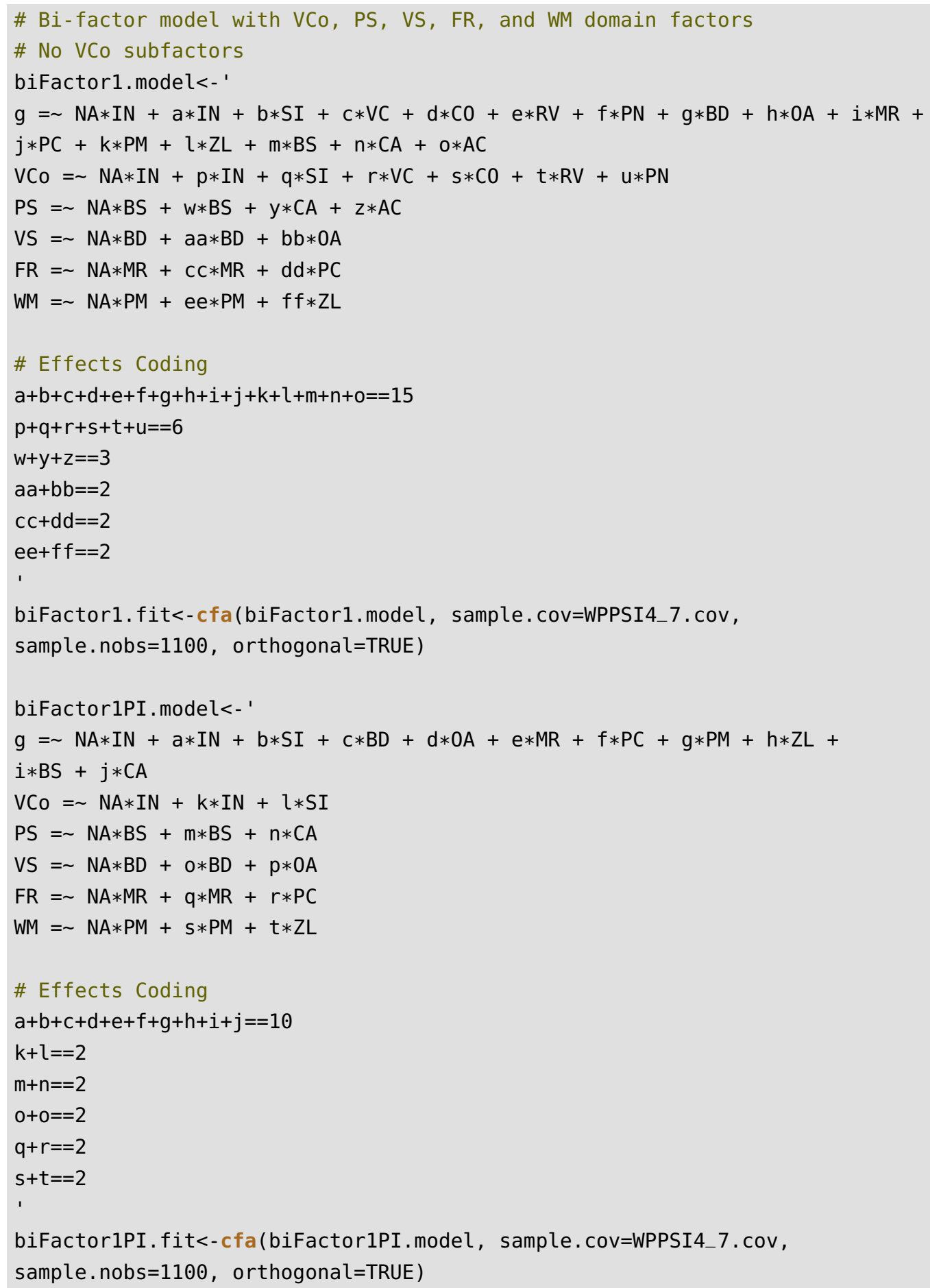




\subsection{Bi-factor model with two Verbal Comprehension subfactors}

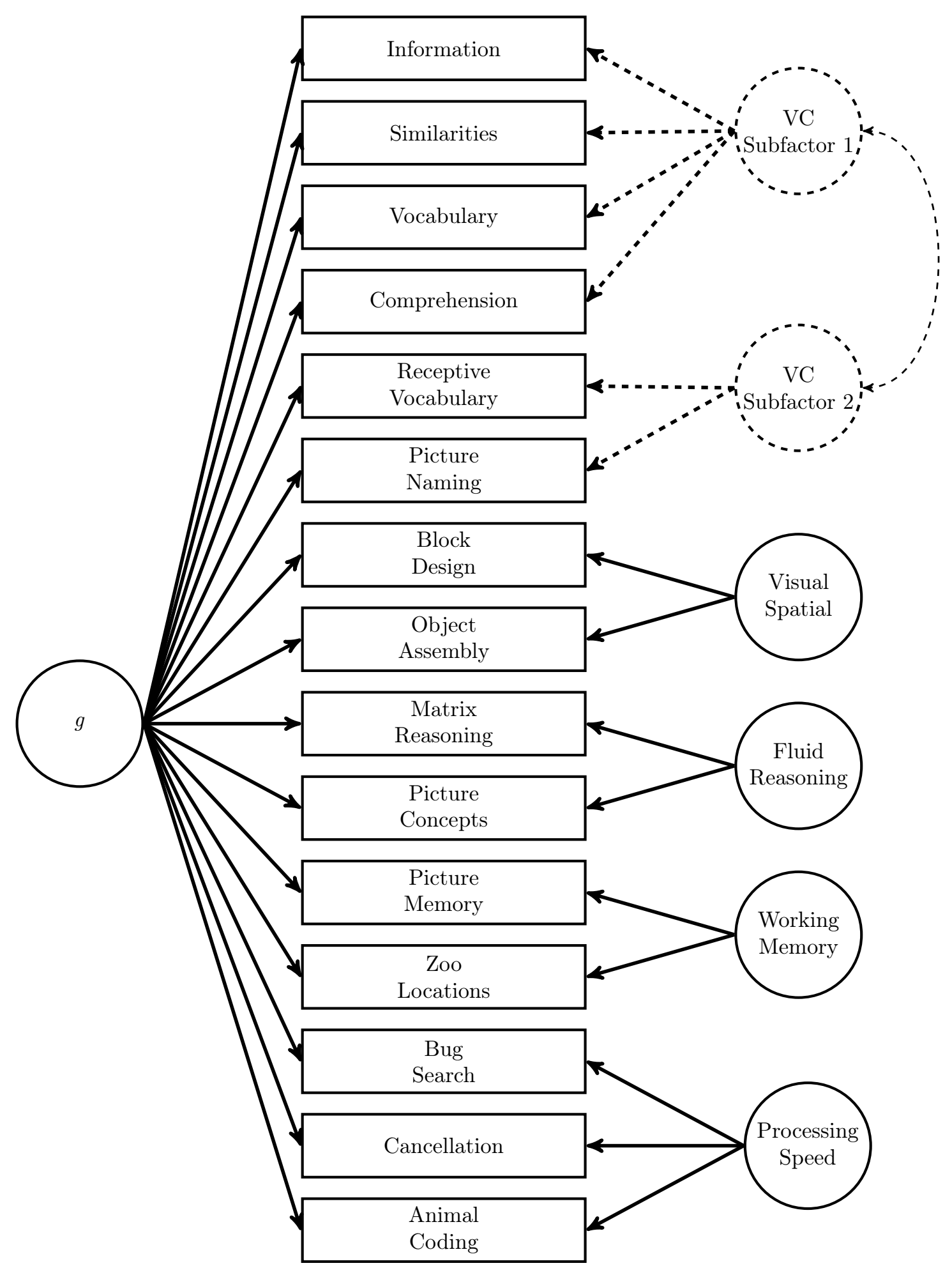

Figure 14: Bi-factor model 2 for 4:0-7:7 year-old group. Residual errors not shown. 


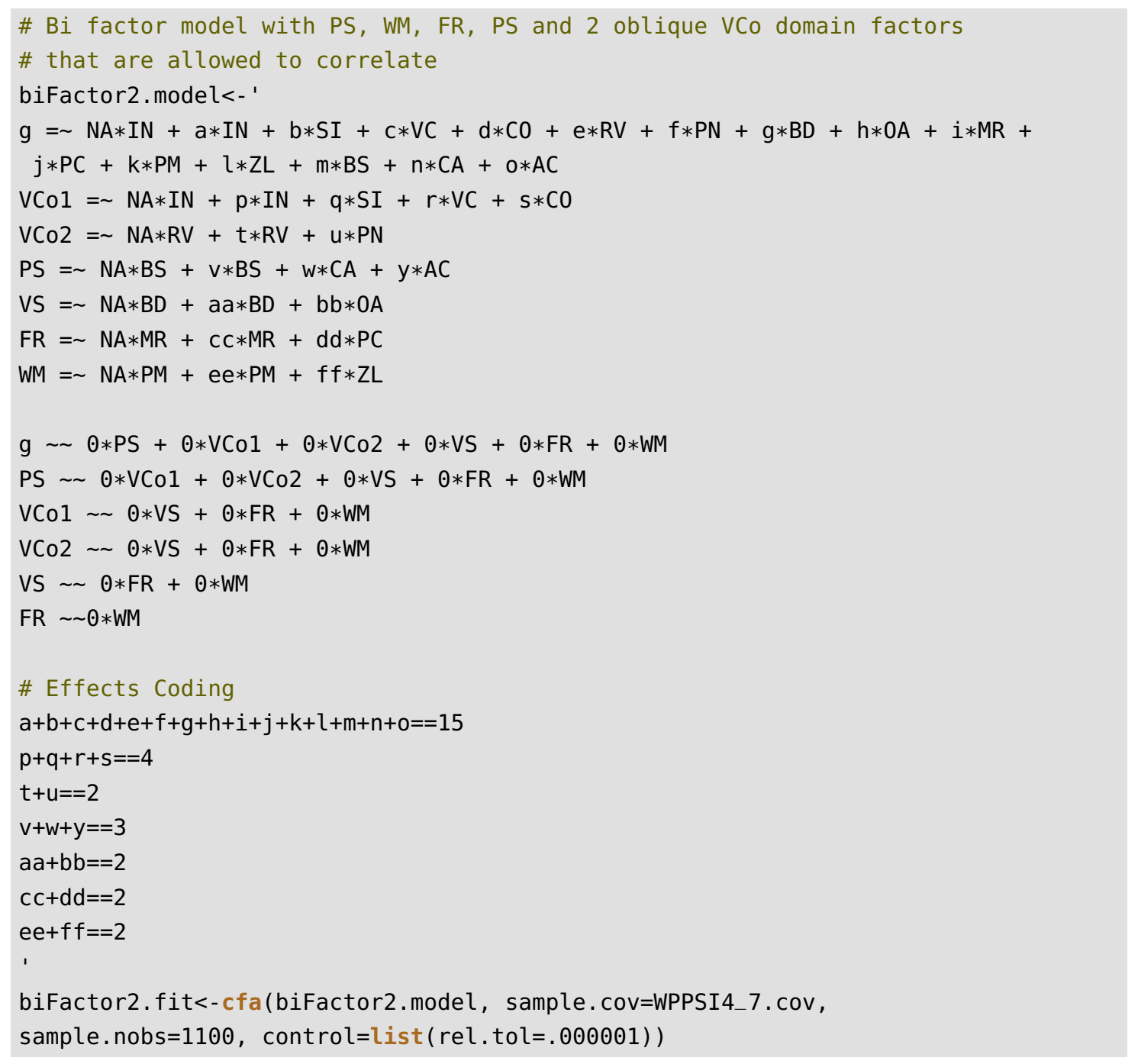




\subsection{No second-order factor and five oblique first-order factors}

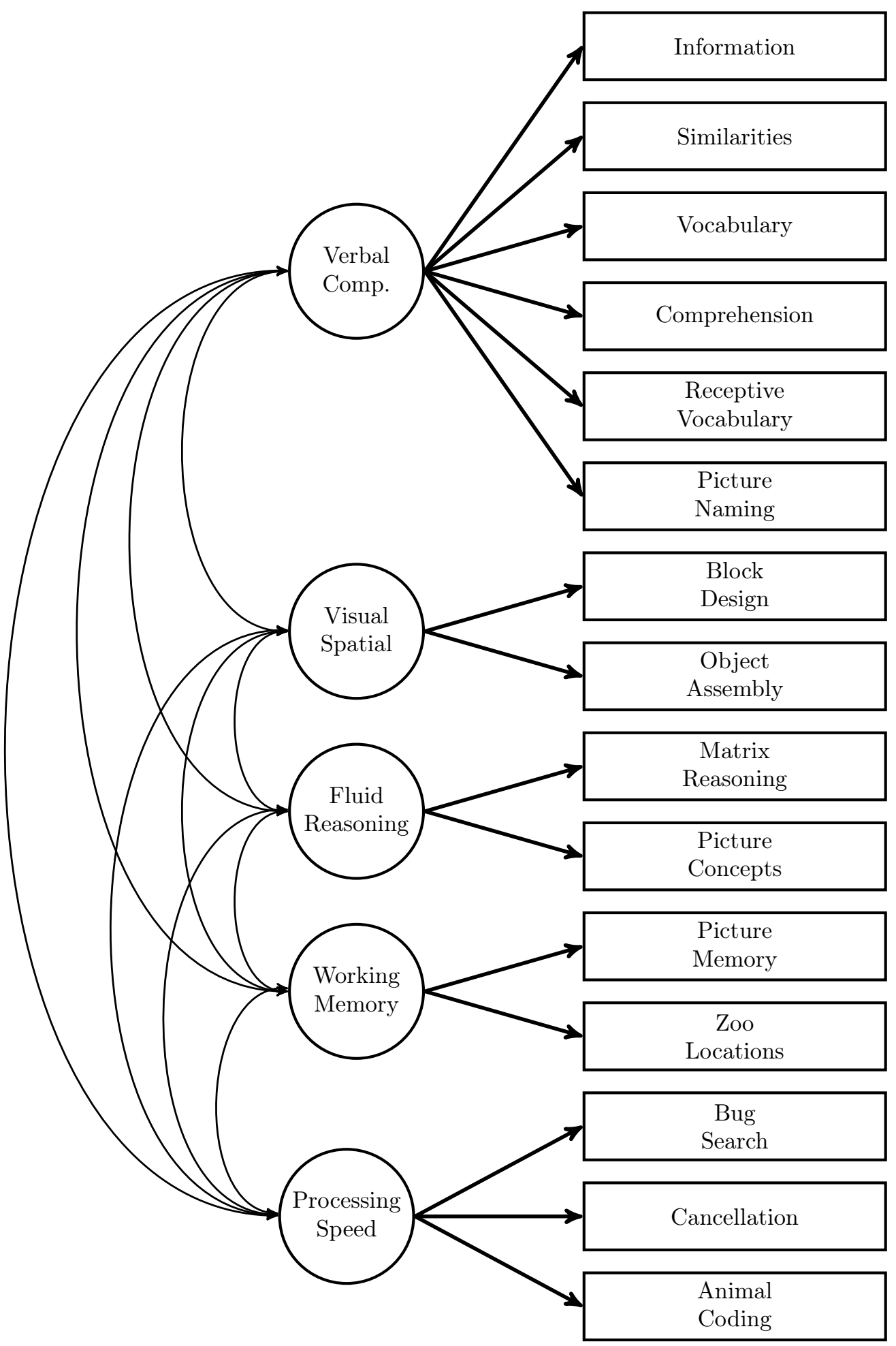

Figure 15: Model with no second-order factors and five oblique first-order factors for 4:0-7:7 year-old group. Residual errors not shown. Correlations among the factors are given in Table D and the variance explained and $\omega$ reliability estimates are given in Table E. 


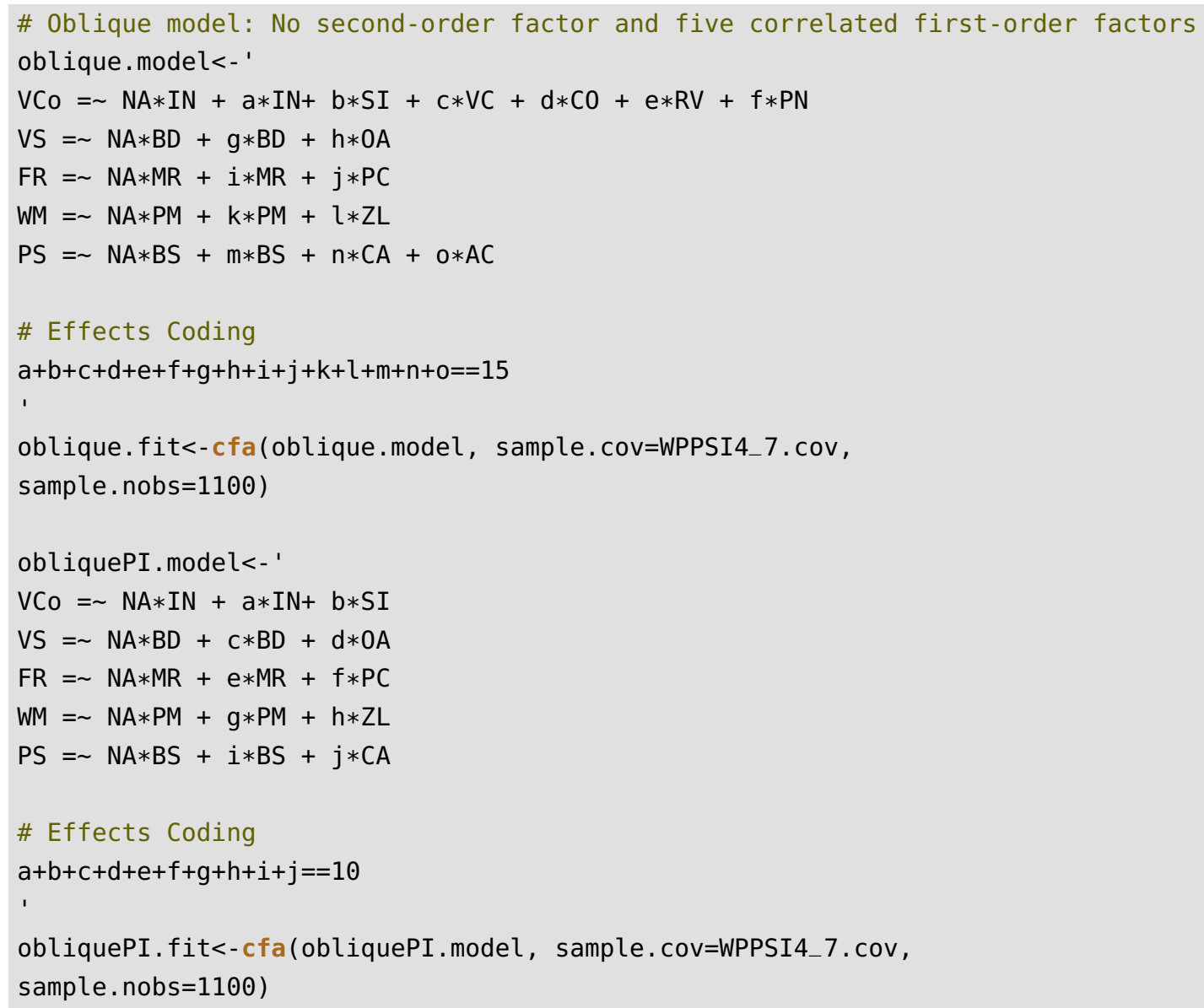

Table D: Correlations among the latent variables for the oblique model for 4:0-7:7 year-old group.

\begin{tabular}{|c|c|c|c|c|c|c|}
\hline \multicolumn{7}{|c|}{ All Subtests } \\
\hline & Subtest & 1 & 2 & 3 & 4 & 5 \\
\hline 1.00 & Verbal Comp. & 1.00 & 0.77 & 0.82 & 0.72 & 0.60 \\
\hline 2.00 & Visual Spatial & 0.77 & 1.00 & 0.88 & 0.81 & 0.67 \\
\hline 3.00 & Fluid Reasoning & 0.82 & 0.88 & 1.00 & 0.87 & 0.69 \\
\hline 4.00 & Working Memory & 0.72 & 0.81 & 0.87 & 1.00 & 0.76 \\
\hline 5.00 & Processing Speed & 0.60 & 0.67 & 0.69 & 0.76 & 1.00 \\
\hline \multicolumn{7}{|c|}{ Primary Index Subtests } \\
\hline & Subtest & 1 & 2 & 3 & 4 & 5 \\
\hline 1.00 & Verbal Comp. & 1.00 & 0.78 & 0.81 & 0.72 & 0.60 \\
\hline 2.00 & Visual Spatial & 0.78 & 1.00 & 0.87 & 0.81 & 0.68 \\
\hline 3.00 & Fluid Reasoning & 0.81 & 0.87 & 1.00 & 0.86 & 0.69 \\
\hline 4.00 & Working Memory & 0.72 & 0.81 & 0.86 & 1.00 & 0.76 \\
\hline 5.00 & Processing Speed & 0.60 & 0.68 & 0.69 & 0.76 & 1.00 \\
\hline
\end{tabular}


Table E: Sources of Variance in the Wechsler Preschool and Primary Scale of Intelligence-Fourth Edition Among 1,100 Children Aged 4:00-7:7 Years for the Oblique Mode.

\begin{tabular}{|c|c|c|c|c|c|c|c|c|c|c|c|c|}
\hline \multirow[b]{3}{*}{ Subtest } & \multirow{2}{*}{\multicolumn{2}{|c|}{$\mathrm{VC}$}} & \multicolumn{8}{|c|}{ Ages 2:6-3:11 Years } & \multirow[b]{3}{*}{$h^{2}$} & \multirow[b]{3}{*}{$u^{2}$} \\
\hline & & & & $\mathrm{S}$ & \multicolumn{2}{|c|}{ WM } & \multicolumn{2}{|c|}{ FR } & \multicolumn{2}{|c|}{ PS } & & \\
\hline & $b$ & Var & $b$ & Var & $b$ & Var & $b$ & Var & $b$ & Var & & \\
\hline IN & 0.80 & 63.52 & & & & & & & & & 63.52 & 36.48 \\
\hline SI & 0.81 & 65.93 & & & & & & & & & 65.93 & 34.07 \\
\hline VO & 0.79 & 61.78 & & & & & & & & & 61.78 & 38.22 \\
\hline $\mathrm{CO}$ & 0.76 & 57.76 & & & & & & & & & 57.76 & 42.24 \\
\hline RV & 0.70 & 48.72 & & & & & & & & & 48.72 & 51.28 \\
\hline $\mathrm{PN}$ & 0.75 & 55.95 & & & & & & & & & 55.95 & 44.05 \\
\hline $\mathrm{BD}$ & & & 0.74 & 54.46 & & & & & & & 54.46 & 45.54 \\
\hline OA & & & 0.69 & 47.75 & & & & & & & 47.75 & 52.25 \\
\hline MR & & & & & & & 0.72 & 51.12 & & & 51.12 & 48.88 \\
\hline $\mathrm{PC}$ & & & & & & & 0.62 & 37.82 & & & 37.82 & 62.18 \\
\hline PM & & & & & 0.68 & 46.10 & & & & & 46.10 & 53.90 \\
\hline ZL & & & & & 0.60 & 36.36 & & & & & 36.36 & 63.64 \\
\hline BS & & & & & & & & & 0.77 & 59.75 & 59.75 & 40.25 \\
\hline $\mathrm{CA}$ & & & & & & & & & 0.60 & 36.36 & 36.36 & 63.64 \\
\hline $\mathrm{AC}$ & & & & & & & & & 0.70 & 48.86 & 48.86 & 51.14 \\
\hline Total & & 23.60 & & 6.80 & & 5.90 & & 5.50 & & 9.70 & & \\
\hline$\omega$ & & 0.90 & & 0.68 & & 0.61 & & 0.58 & & 0.74 & & \\
\hline
\end{tabular}

Note. $b=$ standardized factor loading, Var $=\%$ variance explained, $h^{2}=$ communality, $u^{2}=$ uniqueness, $\mathrm{VC}=$ Verbal Comprehension factor, $\mathrm{VS}=$ Visual-Spatial factor, $\mathrm{WM}=$ Working Memory factor, $\mathrm{PS}=$ Processing Speed factor, FR = Fluid Reasoning factor, IN = Information, RV = Receptive Vocabulary, $\mathrm{PN}=$ Picture Naming, $\mathrm{BD}=$ Block Design, $\mathrm{OA}=$ Object Assembly, $\mathrm{PM}=$ Picture Memory, $\mathrm{ZL}=$ Zoo Locations, $\mathrm{SI}=$ Similarities, $\mathrm{VO}=$ Vocabulary, $\mathrm{CO}=$ Comprehension, $\mathrm{MR}=$ Matrix Reasoning, $\mathrm{PC}=$ Picture Concepts, $\mathrm{BS}=$ Bug Search, $\mathrm{CA}=$ Cancellation, $\mathrm{AC}=$ Animal Coding, $\omega=$ omega. $\omega$ estimated from the omega software program (Watkins, 2013). 
Table F: Fit Statistics for the models used for the 4:0-7:7 year-old group data.

\begin{tabular}{lccccccc}
\hline Model & $\chi^{2}$ & $d f$ & $p$ & CFI & RMSEA & SRMR & AIC \\
\hline \multicolumn{7}{c}{ All Subtests } \\
\hline Model 1 & 950.32 & 90.00 & 0.00 & 0.88 & 0.09 & 0.06 & 76677.51 \\
Model 2 & 500.34 & 86.00 & 0.00 & 0.94 & 0.07 & 0.04 & 76235.54 \\
Model 3 & 284.59 & 84.00 & 0.00 & 0.97 & 0.05 & 0.03 & 76023.79 \\
Model 4a & 270.11 & 82.00 & 0.00 & 0.97 & 0.05 & 0.03 & 76013.30 \\
Model 4b & 263.22 & 82.00 & 0.00 & 0.97 & 0.04 & 0.03 & 76006.42 \\
Model 5a & 249.64 & 80.00 & 0.00 & 0.98 & 0.04 & 0.03 & 75996.84 \\
Model 5b & 212.03 & 76.00 & 0.00 & 0.98 & 0.04 & 0.02 & 75967.23 \\
Bifactor 1 & 231.47 & 75.00 & 0.00 & 0.98 & 0.04 & 0.02 & 75998.66 \\
Bifactor 2 & 191.61 & 74.00 & 0.00 & 0.98 & 0.04 & 0.02 & 75962.81 \\
Oblique & 232.23 & 76.00 & 0.00 & 0.98 & 0.04 & 0.02 & 75987.42 \\
\hline & & Primary Index Subtests & & \\
\hline Model 1 & 310.52 & 35.00 & 0.00 & 0.92 & 0.08 & 0.04 & 51972.36 \\
Model 2 & 500.34 & 86.00 & 0.00 & 0.94 & 0.07 & 0.04 & 76235.54 \\
Model 3 & 104.26 & 29.00 & 0.00 & 0.98 & 0.05 & 0.03 & 51778.10 \\
Model 4a & 91.46 & 27.00 & 0.00 & 0.98 & 0.05 & 0.02 & 51769.30 \\
Model 4b & 85.55 & 27.00 & 0.00 & 0.98 & 0.04 & 0.02 & 51763.39 \\
Model 5a & 70.59 & 25.00 & 0.00 & 0.99 & 0.04 & 0.02 & 51752.43 \\
Bifactor 1 & 70.59 & 25.00 & 0.00 & 0.99 & 0.04 & 0.02 & 51762.43 \\
Oblique & 56.64 & 21.00 & 0.00 & 0.99 & 0.04 & 0.02 & 51746.48 \\
\hline
\end{tabular}

CFI: comparative fit index; RMSEA: root mean square error of approximation; SRMR: standardized root mean square residual; AIC: Akaike's information criterion. 


\section{References}

Beaujean, A. A. (2013). Factor analysis using R. Practical Assessment, Research and Evaluation, $18(4), 1-11$.

Coalson, D. L., \& Raiford, S. E. (2012). Wechsler preschool and primary scale of intelligence technical and Interpretive manual (4th ed.). San Antonio, TX: The Psychological Corporation.

R Development Core Team. (2013). R: A language and environment for statistical computing. Vienna, Austria: R Foundation for Statistical Computing. Available from http://www.R -project.org.

Rosseel, Y. (2012). lavaan: An R package for structural equation modeling. Journal of Statistical Software, $48(2), 1-36$.

Watkins, M. W. (2013). Omega [Computer software]. Phoenix, AZ: Ed \& Psych Associates. 